\title{
Primordial Magnetic Fields from the Post-Inflationary Universe
}

\author{
Takeshi Kobayashi \\ Canadian Institute for Theoretical Astrophysics, University of Toronto, \\ 60 St. George Street, Toronto, Ontario M5S 3H8, Canada \\ Perimeter Institute for Theoretical Physics, \\ 31 Caroline Street North, Waterloo, Ontario N2L 2Y5, Canada \\ E-mail: takeshi@cita.utoronto.ca
}

\begin{abstract}
We explore cosmological magnetogenesis in the post-inflationary universe, when the inflaton oscillates around its potential minimum and the universe is effectively dominated by cold matter. During this epoch prior to reheating, large-scale magnetic fields can be significantly produced by the cosmological background. By considering magnetogenesis both during and after inflation, we demonstrate that magnetic fields stronger than $10^{-15} \mathrm{G}$ can be generated on Mpc scales without having strong couplings in the theory, or producing too large electric fields that would dominate the universe.
\end{abstract}




\section{Contents}

1 Introduction $\quad 1$

2 Electromagnetic Fields in an Expanding Universe $\quad 2$

2.1 Quantization of Electromagnetic Fields . . . . . . . . . . . . . . . 3

2.2 Power Spectra of Magnetic and Electric Fields . . . . . . . . . . . . . . . 5

2.3 Evolution of Mode Functions . . . . . . . . . . . . . . . . . 6

3 Magnetogenesis During Inflation $\quad 7$

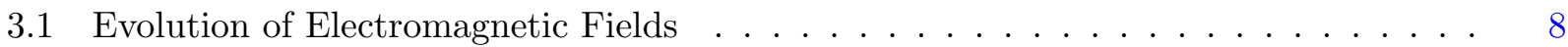

3.2 Constraints on Inflation $\ldots \ldots \ldots \ldots$

4 Magnetogenesis After Inflation $\quad 13$

4.1 Evolution of Electromagnetic Fields . . . . . . . . . . . . . . . . . 13

4.2 Constraints on Reheating . . . . . . . . . . . . . . . . . . . . 17

5 Large Magnetic Fields from Two Step Magnetogenesis $\quad 18$

5.1 Magnetic Fields and Energy Bounds . . . . . . . . . . . . . . . . 18

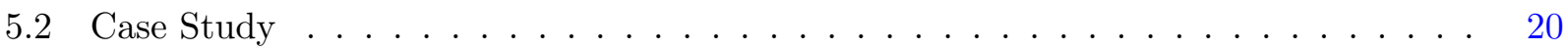

6 Examples of Scalar Couplings $\quad 23$

6.1 Couplings During Inflation . . . . . . . . . . . . . . . . . . . . 24

6.2 Couplings After Inflation . . . . . . . . . . . . . . . . 25

$\begin{array}{llr}7 & \text { Conclusions } & 26\end{array}$

\section{$1 \quad$ Introduction}

Large-scale magnetic fields exist in cosmic structures such as galaxies and galaxy clusters, however the origin of the fields remain unexplained. Recent gamma ray observations suggest the existence of magnetic fields even in void regions $[1,2,3,4,5,6]$, and the amplitude of such intergalactic magnetic fields were derived to be stronger than $\sim 10^{-15} \mathrm{G}$. Although this lower bound has astrophysical uncertainties (see e.g. [7, 8]), such large-scale magnetic fields hint that the magnetic fields are of cosmological origin.

Here it should be noted that the equations of motion of the electromagnetic fields in a Friedmann universe reduce to those in Minkowski space, since the standard Maxwell theory is conformally invariant. Therefore the conformal invariance should be broken for the electromagnetic fields to be significantly produced by the cosmological background. Such mechanisms of cosmological magnetogenesis have been embedded in the inflationary universe in various works, e.g., $[9,10,11,12,13,14$, $15,16,17,18] .{ }^{1}$ However the studies have revealed that inflationary magnetogenesis cannot produce

\footnotetext{
${ }^{1}$ As an alternative way of cosmologically producing magnetic fields, $[19,20,21]$ have studied magnetogenesis during phase transitions.
} 
significant magnetic fields without running into at least one of the inconsistencies: (i) the electromagnetic fields obtain strong couplings and therefore the theory becomes uncontrollable [12, 17, 22], (ii) too large electric fields are produced such that their backreaction can spoil inflation and/or magnetogenesis $[15,17,18,22]$. In particular, the backreaction of the electric fields is a problem inherent to inflationary magnetogenesis; after magnetogenesis the magnetic fields decay as $B \propto a^{-2}$ where $a$ is the scale factor, therefore the present day magnetic fields should be remnants of substantial photon production during a short period of time in the very early universe. In other words, the electromagnetic vector potential during inflation is required to possess a large time-derivative, and therefore leads to excessive production of electric fields. Recently it has also been pointed out that the generated electromagnetic fields can induce cosmological density perturbations beyond the observed value, and works $[23,24,25,26,27]$ have imposed further constraints on inflationary magnetogenesis. (See also $[28,29,30,31]$ and references therein for constraints on primordial magnetic fields from CMB and large-scale structure data.) However, we stress that the inflationary expansion itself is not a necessary condition for magnetogenesis; it is the breaking of the conformal invariance that allows magnetic field production by the cosmological background.

In this paper, we investigate post-inflationary magnetogenesis by breaking the conformal invariance of the Maxwell theory after inflation. The conductivity of the universe becomes high during reheating and thereafter the magnetic fields decay as $B \propto a^{-2}$ [9]. Therefore we focus on the epoch between the end of inflation and (p)reheating, during which the inflaton field oscillates around its potential minimum and the universe is effectively dominated by cold matter. The conformal invariance of the Maxwell theory is broken by couplings between the electromagnetic fields and scalar degrees of freedom which can be the inflaton or some other spectator field(s). By considering magnetogenesis both during and after inflation, we demonstrate that magnetic fields of $10^{-15} \mathrm{G}$ or stronger can be produced at cosmological scales (say, on Mpc scales), without running into the strong coupling regime or producing too much electric fields. The problem of affecting the cosmological density perturbations is also ameliorated since the magnetic fields are enhanced after inflation. The proposed model is compatible even with high scale inflation.

This paper is organized as follows. We first review the electromagnetic theory in an expanding universe in Section 2. Then we move on to study magnetogenesis during the inflationary epoch in Section 3, and post-inflationary magnetogenesis in Section 4. Here we will see that the magnetogenesis in each epoch alone are highly constrained by the strong coupling and backreaction problems. In particular, the constraints on inflationary and post-inflationary magnetogenesis will be translated into severe upper bounds on the inflation and reheating scales, respectively. Then in Section 5 we discuss the combined scenario of inflationary and post-inflationary magnetogenesis. There we will see that such a two step model can overcome the challenges and efficiently produce large-scale mag-

netic fields. In Section 6, we present some examples of scalar couplings that break the conformal invariance of the Maxwell theory. Finally, we conclude in Section 7.

\section{Electromagnetic Fields in an Expanding Universe}

Let us start by reviewing the evolution of electromagnetic fields in an expanding universe. For a more detailed review see $[32,33,34]$. 
Throughout this paper we study an electromagnetic theory with an effective coupling $I$,

$$
S=\int d^{4} x \sqrt{-g}\left(-\frac{I^{2}}{4} F_{\mu \nu} F^{\mu \nu}\right)
$$

where $F_{\mu \nu}=\partial_{\mu} A_{\nu}-\partial_{\nu} A_{\mu}$ and the Greek indices take values $\mu, \nu=0,1,2,3$. The coupling $I$ is an arbitrary function of other degrees of freedom, for instance, of a scalar field, $I=I(\sigma)[10]$. The standard Maxwell theory is recovered when $I$ is a constant, then the theory is invariant under the conformal transformation $g_{\mu \nu} \rightarrow \Omega^{2} g_{\mu \nu}{ }^{2}$

It should also be noted that $I$ being tiny, i.e. $I^{2} \ll 1$, can lead to strong couplings in the electromagnetic theory. Considering an interaction term with a charged fermion such as $c \bar{\psi} \gamma^{\mu} \psi A_{\mu}$ where $c$ is a coupling constant, then after canonically normalizing the field as $\tilde{A}_{\mu}=I A_{\mu}$, the interaction term becomes $\frac{c}{I} \bar{\psi} \gamma^{\mu} \psi \tilde{A}_{\mu}$ and one sees that the effective coupling is $c / I$. Thus a tiny $I$ would push the theory into the strong coupling regime where perturbative calculations break down.

\subsection{Quantization of Electromagnetic Fields}

We consider a flat FRW background

$$
d s^{2}=g_{\mu \nu} d x^{\mu} d x^{\nu}=a(\tau)^{2}\left(-d \tau^{2}+d \boldsymbol{x}^{2}\right)
$$

where $\tau$ is the conformal time. The coupling $I$ is also considered to be homogeneous, i.e., $I=I(\tau)$. (In case where $I$ is a function of a scalar field $\sigma$, then we suppose $\sigma=\sigma(\tau)$.) We decompose the spatial components of the vector potential $A_{i}$ into irrotational and incompressible parts,

$$
A_{\mu}=\left(A_{0}, \partial_{i} S+V_{i}\right)
$$

where

$$
\partial_{i} V_{i}=0
$$

The Latin letters denote spatial indices $i, j=1,2,3$, and the sum over repeated spatial indices is implied irrespective of their positions. Then the action (2.1) can be rewritten as, up to total derivatives,

$$
S=\int d \tau d^{3} x \frac{I^{2}}{2}\left\{V_{i}^{\prime} V_{i}^{\prime}+\partial_{i} S^{\prime} \partial_{i} S^{\prime}+\partial_{i} A_{0} \partial_{i} A_{0}-2 \partial_{i} S^{\prime} \partial_{i} A_{0}-\partial_{i} V_{j} \partial_{i} V_{j}\right\}
$$

where a prime denotes a $\tau$-derivative. Varying the action in terms of the Lagrange multiplier $A_{0}$ and choosing proper boundary conditions, we obtain

$$
A_{0}=S^{\prime}
$$

\footnotetext{
${ }^{2}$ Alternatively, the conformal invariance of the electromagnetic fields can be broken by a photon mass term $m_{\gamma}^{2} A_{\mu} A^{\mu}$. However significant magnetogenesis requires a tachyonic mass $m_{\gamma}^{2}<0$, which can arise from, e.g., non-minimal couplings between the electromagnetic field and gravity [9]. However such a theory contains several problems including the existence of a ghost $[35,36]$. (See also discussions in [17, 22].) Therefore in this paper we focus on massless photons whose conformal invariance is broken through the kinetic coupling (2.1).
} 
By substituting this constraint into the action (2.5), the scalar modes $A_{0}$ and $S$ vanish and we are left with the two polarization states of the photon (note the constraint (2.4)),

$$
S=\int d \tau d^{3} x \frac{I^{2}}{2}\left\{V_{i}^{\prime} V_{i}^{\prime}-\partial_{i} V_{j} \partial_{i} V_{j}\right\}
$$

The equation of motion of $V_{i}$ reads

$$
V_{i}^{\prime \prime}+2 \frac{I^{\prime}}{I} V_{i}^{\prime}-\partial^{2} V_{i}=0
$$

where $\partial^{2} \equiv \partial_{j} \partial_{j}$. Now considering the Fourier modes,

$$
V_{i}(\tau, \boldsymbol{x})=\frac{1}{(2 \pi)^{3}} \int d^{3} k e^{i \boldsymbol{k} \cdot \boldsymbol{x}} \xi_{i}(\tau, \boldsymbol{k})
$$

then $\xi_{i} k_{i}=0$ should be satisfied from the constraint (2.4). Let us express $\xi_{i}$ as a linear combination of two orthonormal polarization vectors $\epsilon_{i}^{(p)}(\boldsymbol{k})$ with $p=1,2$, satisfying

$$
\epsilon_{i}^{(p)}(\boldsymbol{k}) k_{i}=0, \quad \epsilon_{i}^{(p)}(\boldsymbol{k}) \epsilon_{i}^{(q)}(\boldsymbol{k})=\delta_{p q}
$$

Note that from (2.10) follows

$$
\sum_{p=1,2} \epsilon_{i}^{(p)}(\boldsymbol{k}) \epsilon_{j}^{(p)}(\boldsymbol{k})=\delta_{i j}-\frac{k_{i} k_{j}}{k^{2}}
$$

where $k \equiv|\boldsymbol{k}|$. Unlike the spacetime indices, we do not assume implicit summation over the polarization index $(p)$ throughout this paper.

Let us now quantize the theory by promoting $V_{i}(2.9)$ to an operator,

$$
V_{i}(\tau, \boldsymbol{x})=\frac{1}{(2 \pi)^{3}} \sum_{p=1,2} \int d^{3} k \epsilon_{i}^{(p)}(\boldsymbol{k})\left\{e^{i \boldsymbol{k} \cdot \boldsymbol{x}} a_{\boldsymbol{k}}^{(p)} u_{\boldsymbol{k}}^{(p)}(\tau)+e^{-i \boldsymbol{k} \cdot \boldsymbol{x}} a_{\boldsymbol{k}}^{\dagger(p)} u_{\boldsymbol{k}}^{*(p)}(\tau)\right\} .
$$

Here $a_{\boldsymbol{k}}^{(p)}$ and $a_{\boldsymbol{k}}^{\dagger(p)}$ are annihilation and creation operators, respectively, and $u_{\boldsymbol{k}}^{(p)}(\tau)$ is a mode function that satisfies the equation of motion (cf. (2.8)),

$$
u_{\boldsymbol{k}}^{\prime \prime(p)}+2 \frac{I^{\prime}}{I} u_{\boldsymbol{k}}^{(p)}+k^{2} u_{\boldsymbol{k}}^{(p)}=0 .
$$

Here it should be noted that the time evolving coupling $I$ gives rise to a (positive or negative) friction term; the standard Maxwell theory with $I=$ const. brings the equation of motion to the wave equation in a flat spacetime.

The conjugate momentum of $V_{i}$ is obtained from the action $S=\int d \tau d^{3} x \mathcal{L}$ in (2.7) as

$$
\Pi^{i}=\frac{\partial \mathcal{L}}{\partial V_{i}^{\prime}}=I^{2} V_{i}^{\prime}
$$

and the commutation relations are imposed as

$$
\left[a_{\boldsymbol{k}}^{(p)}, a_{\boldsymbol{h}}^{(q)}\right]=\left[a_{\boldsymbol{k}}^{\dagger(p)}, a_{\boldsymbol{h}}^{\dagger(q)}\right]=0, \quad\left[a_{\boldsymbol{k}}^{(p)}, a_{\boldsymbol{h}}^{\dagger(q)}\right]=(2 \pi)^{3} \delta^{p q} \delta^{(3)}(\boldsymbol{k}-\boldsymbol{h}),
$$




$$
\begin{gathered}
{\left[V_{i}(\tau, \boldsymbol{x}), V_{j}(\tau, \boldsymbol{y})\right]=\left[\Pi^{i}(\tau, \boldsymbol{x}), \Pi^{j}(\tau, \boldsymbol{y})\right]=0,} \\
{\left[V_{i}(\tau, \boldsymbol{x}), \Pi^{j}(\tau, \boldsymbol{y})\right]=i \delta^{(3)}(\boldsymbol{x}-\boldsymbol{y})\left(\delta_{i j}-\frac{\partial_{i} \partial_{j}}{\partial^{2}}\right) .}
\end{gathered}
$$

Using (2.11), the relation (2.17) can be rewritten as

$$
\left[V_{i}(\tau, \boldsymbol{x}), \Pi^{j}(\tau, \boldsymbol{y})\right]=\frac{i}{(2 \pi)^{3}} \sum_{p=1,2} \int d^{3} k e^{i \boldsymbol{k} \cdot(\boldsymbol{x}-\boldsymbol{y})} \epsilon_{i}^{(p)}(\boldsymbol{k}) \epsilon_{j}^{(p)}(\boldsymbol{k}) .
$$

Choosing the polarization vectors such that $\epsilon_{i}^{(p)}(\boldsymbol{k})=\epsilon_{i}^{(p)}(-\boldsymbol{k})$, then one can check that the commutation relations (2.16) and (2.18) follow from (2.15) when the mode function is independent of the direction of $\boldsymbol{k}$, i.e.,

$$
u_{\boldsymbol{k}}^{(p)}=u_{k}^{(p)}
$$

and obeys the normalization condition

$$
I^{2}\left(u_{k}^{(p)} u_{k}^{*(p)}-u_{k}^{*(p)} u_{k}^{\prime(p)}\right)=i
$$

\subsection{Power Spectra of Magnetic and Electric Fields}

The magnetic and electric fields measured by a comoving observer with 4 -velocity $u^{\mu}\left(u^{i}=0\right.$, $\left.u_{\mu} u^{\mu}=-1\right)$ is

$$
B_{\mu}=\frac{1}{2} \varepsilon_{\mu \nu \sigma} F^{\nu \sigma}, \quad E_{\mu}=u^{\nu} F_{\mu \nu}
$$

where

$$
\varepsilon_{\mu \nu \sigma}=\eta_{\mu \nu \sigma \lambda} u^{\lambda}
$$

and $\eta_{\mu \nu \sigma \lambda}$ is a totally antisymmetric permutation tensor with $\eta_{0123}=-\sqrt{-g}$. The time-components $B_{0}$ and $E_{0}$ vanish, and we find

$$
B_{i}=\frac{1}{a^{4}} \varepsilon_{i j k} \partial_{j} A_{k}, \quad E_{i}=-\frac{1}{a} V_{i}^{\prime} .
$$

Note that $\varepsilon_{i j k}$ is totally antisymmetric with $\varepsilon_{123}=a^{3}$. Hence the magnitude of the fields are given by

$$
\begin{aligned}
B^{2} \equiv B_{\mu} B^{\mu} & =\frac{1}{a^{2}} B_{i} B_{i}=\frac{1}{a^{4}}\left(\partial_{i} V_{j} \partial_{i} V_{j}-\partial_{i} V_{j} \partial_{j} V_{i}\right) \\
E^{2} & \equiv E_{\mu} E^{\mu}=\frac{1}{a^{2}} E_{i} E_{i}=\frac{1}{a^{4}} V_{i}^{\prime} V_{i}^{\prime}
\end{aligned}
$$

Choosing the vacuum as $a_{\boldsymbol{k}}^{(p)}|0\rangle=0$ for $p=1,2$ and ${ }^{\forall} \boldsymbol{k}$, then from (2.12) and the commutation relation (2.15), we obtain the correlation functions

$$
\begin{aligned}
\left\langle B_{\mu}(\tau, \boldsymbol{x}) B^{\mu}(\tau, \boldsymbol{y})\right\rangle & =\int \frac{d^{3} k}{4 \pi k^{3}} e^{i \boldsymbol{k} \cdot(\boldsymbol{x}-\boldsymbol{y})} \mathcal{P}_{B}(\tau, k), \\
\left\langle E_{\mu}(\tau, \boldsymbol{x}) E^{\mu}(\tau, \boldsymbol{y})\right\rangle & =\int \frac{d^{3} k}{4 \pi k^{3}} e^{i \boldsymbol{k} \cdot(\boldsymbol{x}-\boldsymbol{y})} \mathcal{P}_{E}(\tau, k),
\end{aligned}
$$


where the power spectra are expressed in terms of the mode functions as

$$
\begin{aligned}
& \mathcal{P}_{B}(\tau, k)=\frac{k^{5}}{2 \pi^{2} a(\tau)^{4}} \sum_{p=1,2}\left|u_{k}^{(p)}(\tau)\right|^{2}, \\
& \mathcal{P}_{E}(\tau, k)=\frac{k^{3}}{2 \pi^{2} a(\tau)^{4}} \sum_{p=1,2}\left|u_{k}^{\prime(p)}(\tau)\right|^{2} .
\end{aligned}
$$

Furthermore, the energy-momentum tensor of the electromagnetic fields is

$$
T_{\mu \nu}^{\mathrm{EM}}=I^{2}\left(F_{\mu \sigma} F_{\nu}^{\sigma}-\frac{1}{4} g_{\mu \nu} F_{\sigma \lambda} F^{\sigma \lambda}\right)
$$

and the comoving observer measures the energy density as

$$
T_{\mu \nu}^{\mathrm{EM}} u^{\mu} u^{\nu}=-T_{0}^{0}=\frac{I^{2}}{2}\left(B^{2}+E^{2}\right)
$$

Hence the energy density of the electromagnetic fields are expressed in terms of the power spectra (2.28) and (2.29) as

$$
\rho_{\mathrm{EM}}=\left\langle-T_{0}^{0}(\tau, \boldsymbol{x})\right\rangle=\frac{I^{2}}{2} \int \frac{d k}{k}\left(\mathcal{P}_{B}+\mathcal{P}_{E}\right)
$$

which clearly shows the contribution from each of the magnetic and electric fields.

\subsection{Evolution of Mode Functions}

Let us now see how the mode function $u_{k}^{(p)}$ evolves as the universe expands. We suppose a flat FRW background (2.2) with an equation of state parameter that takes a constant value except for $-1 / 3$,

$$
\frac{p_{\mathrm{bg}}}{\rho_{\mathrm{bg}}}=w=\text { const. } \neq-\frac{1}{3} \text {. }
$$

Then the conformal time is expressed as

$$
d \tau=\frac{2}{1+3 w} d\left(\frac{1}{a H}\right)
$$

in terms of the Hubble parameter $H=a^{\prime} / a^{2}=\dot{a} / a$, where an overdot denotes a derivative with respect to the cosmological time $d t=a d \tau$.

Here we consider a coupling $I$ that scales as a power-law of the scale factor, i.e.,

$$
I \propto a^{-s}
$$

with a constant $s$. Then the electromagnetic fields experience an effective "horizon" with radius of $\sim|I / \dot{I}|=(|s| H)^{-1}$ (see the equation of motion (2.13)), which is similar in size to the Hubble horizon up to the factor $s$. We mainly focus on this case in the following sections, and explicit realizations of such scaling behaviors through couplings with scalar fields are discussed in Section 6 .

Then the general solution for the mode function $u_{k}^{(p)}$ in (2.13) is given by Hankel functions multiplied by powers of $a H$,

$$
(a H)^{-\alpha} H_{\alpha}^{(1),(2)}\left(\frac{2}{|1+3 w|} \frac{k}{a H}\right) \quad \text { with } \quad \alpha=\frac{1}{2}+\frac{2 s}{1+3 w} .
$$


In the sub-horizon limit, i.e. $k / a H \rightarrow \infty$, the solutions (2.36) are approximated by

$$
\begin{aligned}
(a H)^{-\alpha} H_{\alpha}^{(1)} & \left(\frac{2}{|1+3 w|} \frac{k}{a H}\right) \\
& \simeq(a H)^{-\alpha}\left(\frac{|1+3 w|}{\pi} \frac{a H}{k}\right)^{1 / 2} \exp \left\{i\left(\frac{2}{|1+3 w|} \frac{k}{a H}-\frac{2 \alpha+1}{4} \pi\right)\right\}
\end{aligned}
$$

and its complex conjugate for $(a H)^{-\alpha} H_{\alpha}^{(2)}$.

On the other hand, in the super-horizon limit, i.e. $k / a H \rightarrow 0$, each of the two solutions in (2.36) asymptote to a linear combination of the following terms:

$$
\begin{aligned}
& (a H)^{-2 \alpha} \text {, const. for } \alpha \neq 0 \text {, } \\
& \ln (a H), \quad \text { const. for } \quad \alpha=0 \text {. }
\end{aligned}
$$

In other words, $u_{k}^{(p)}$ outside the horizon consists of a time dependent mode and a constant mode. These sets of super-horizon solutions can also be obtained directly from the equation of motion (2.13) by omitting the $k^{2} u_{k}^{(p)}$ term, which gives the solutions

$$
\int^{\tau} \frac{d \tau}{I^{2}}, \text { const. }
$$

The standard Maxwell theory corresponds to the case of $s=0$, i.e. $\alpha=1 / 2$. Then the equation of motion (2.13) reduces to that in Minkowski space, and $u_{k}^{(p)}$ is a sum of plane waves $e^{ \pm i k \tau}{ }^{3}$ Taking the positive frequency solution, we can set its normalization by the condition (2.20) and choose

$$
u_{k}^{(p)}=\frac{e^{-i k \tau}}{(2 k)^{1 / 2} I} .
$$

In this case the powers of the electromagnetic fields are

$$
\mathcal{P}_{B}=\mathcal{P}_{E}=\frac{k^{4}}{2 \pi^{2} I^{2} a^{4}},
$$

which redshift as $\propto a^{-4}$, and the spectra have a blue tilt of $d \ln \mathcal{P}_{B(E)} / d \ln k=4$.

\section{$3 \quad$ Magnetogenesis During Inflation}

Based on the discussions in the previous section, we now examine magnetogenesis in the early universe. We will especially focus on whether the produced magnetic field strengths reach values inferred from gamma ray experiments that have observed $\mathrm{TeV}$ gamma ray emitting blazars: The emitted $\mathrm{TeV}$-scale gamma rays interact with the extragalactic background light and emit pairs of electrons and positrons. The produced electron positron pairs then inverse Compton scatter off

\footnotetext{
${ }^{3}$ The super-horizon solution (2.38) with $\alpha=1 / 2$ holds a growing or decaying mode $\propto(a H)^{-1}$, in addition to the constant solution. This can also be seen by expanding the plane waves up to linear order in $k \tau$ as $\sin (k \tau) \simeq k \tau$, $\cos (k \tau) \simeq 1$. (See also the relation between $\tau$ and $a H$ in (2.34).)
} 
CMB photons, producing an electromagnetic cascade. Since magnetic fields affect the trajectories of electrons and positrons, the (non-)detection of the secondary cascade gamma rays can be used to measure the strength of intergalactic magnetic fields. Works such as $[1,2,3,4,5,6]$ have derived lower bounds on the magnetic fields along the line of sight towards the blazars to be of $\sim 10^{-15} \mathrm{G}$, with an uncertainty of a few orders. The correlation length of the intergalactic magnetic field is considered to be larger than Mpc scales, as the electron positron pairs lose energy on inverse Compton scattering on distance scales of order Mpc. If the magnetic field correlation length is much smaller than a Mpc, then even stronger magnetic fields would be required to explain the observational results. In the following discussions, to be specific, we aim to generate magnetic fields with amplitude $\mathcal{P}_{B}^{1 / 2} \sim 10^{-15} G$ on Mpc scales in the present universe.

We start by discussing magnetogenesis during inflation in this section. As it has already been shown in previous works, we will see that inflationary magnetogenesis is strongly constrained by the strong coupling and backreaction problems. We then investigate post-inflationary magnetogenesis in Section 4, and in Section 5 a successful magnetogenesis model is presented. Throughout the discussions we focus on kinetic couplings $I$ that give rise to an effective "horizon" for a finite period of time, during which the quantum fluctuations of the magnetic fields are enhanced. We also consider the coupling to scale as a power-law of the scale factor.

\subsection{Evolution of Electromagnetic Fields}

Let us consider an inflating background with $H=H_{\text {inf }}=$ const., i.e. $w=-1$. As for the coupling, we assume that it scales as a power-law of the scale factor until a certain time $a=a_{1}$, then $I$ approaches a constant value $I_{1}(>0)$,

$$
I= \begin{cases}I_{1}\left(\frac{a_{1}}{a}\right)^{s} & \text { for } a \leq a_{1}, \\ I_{1} & \text { for } a>a_{1} .\end{cases}
$$

Such a behavior can be realized by electromagnetic fields coupled to, e.g., an inflaton or a spectator field, as is discussed in Section 6.1. The dynamical $I$ generates the effective horizon whose size is similar to that of the Hubble horizon, up to the factor of $s$. In order for efficient magnetogenesis to happen, we suppose the power to be

$$
s>\frac{1}{2},
$$

so that the electromagnetic mode function possesses a growing mode outside the horizon, see (2.38).

\subsection{1 $a \leq a_{1}$}

The mode function $u_{k}$ (Hereafter we omit the polarization index $(p)$ as the discussions are indepen-

dent of the polarization state.) during $a \leq a_{1}$ takes the solution $\left(a H_{\text {inf }}\right)^{s-\frac{1}{2}} H_{-s+\frac{1}{2}}^{(1)}$ which asymptotes to the positive frequency solution in the sub-horizon limit $k \gg a H_{\text {inf }}$, cf. (2.37). Setting the normalization from (2.20), we obtain

$$
u_{k}=\frac{1}{2 I}\left(\frac{\pi}{a H_{\mathrm{inf}}}\right)^{1 / 2} H_{-s+\frac{1}{2}}^{(1)}\left(\frac{k}{a H_{\mathrm{inf}}}\right),
$$


up to an arbitrary phase. When the mode is well inside the horizon, i.e. $k \gg a H_{\text {inf }}$, the mode function is approximated by

$$
u_{k} \simeq \frac{1}{(2 k)^{1 / 2} I} \exp \left\{i\left(\frac{k}{a H_{\mathrm{inf}}}+\frac{s-1}{2} \pi\right)\right\}
$$

whose amplitude evolves as $\left|u_{k}\right| \propto a^{s}$. In the super-horizon limit, i.e. $k \ll a H_{\text {inf }}$, the mode function (3.3) asymptotes to

$$
u_{k} \simeq \frac{e^{i\left(s-\frac{1}{2}\right) \pi}}{I}\left(\frac{\pi}{2 k}\right)^{1 / 2}\left\{\frac{1}{\Gamma\left(s+\frac{1}{2}\right)}\left(\frac{2 a H_{\mathrm{inf}}}{k}\right)^{-s}-i \frac{\Gamma\left(s-\frac{1}{2}\right)}{\pi}\left(\frac{2 a H_{\mathrm{inf}}}{k}\right)^{s-1}\right\} .
$$

Together with the time dependent $I$ in the prefactor, the first term inside the \{\} parentheses gives a constant mode, while the second term gives the growing mode $\propto a^{2 s-1}$. We should remark that

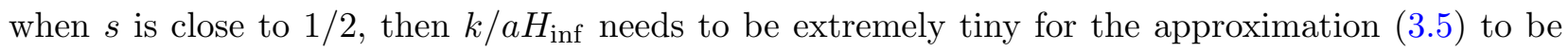
valid. In the following discussions, we suppose that $s$ is not too close to $1 / 2$ and use (3.5) for wave modes outside the horizon.

Using the above approximations, the power spectra of the magnetic (2.28) and electric (2.29) fields are obtained as,

$$
\begin{aligned}
& \mathcal{P}_{B} \simeq \begin{cases}\frac{k^{4}}{2 \pi^{2} I^{2} a^{4}} \frac{\Gamma\left(s-\frac{1}{2}\right)^{2}}{\pi}\left(\frac{2 a H_{\mathrm{inf}}}{k}\right)^{2(s-1)} & \text { for } k \ll a H_{\mathrm{inf}}, \\
\frac{k^{4}}{2 \pi^{2} I^{2} a^{4}} & \text { for } k \gg a H_{\mathrm{inf}},\end{cases} \\
& \mathcal{P}_{E} \simeq \begin{cases}\frac{k^{4}}{2 \pi^{2} I^{2} a^{4}} \frac{\Gamma\left(s+\frac{1}{2}\right)^{2}}{\pi}\left(\frac{2 a H_{\mathrm{inf}}}{k}\right)^{2 s} & \text { for } k \ll a H_{\mathrm{inf}}, \\
\frac{k^{4}}{2 \pi^{2} I^{2} a^{4}} & \text { for } k \gg a H_{\mathrm{inf}} .\end{cases}
\end{aligned}
$$

From these power spectra, we can further compute the energy density of the electromagnetic fields. Contributions to the energy density from sub-horizon modes are renormalized, hence we take the upper limit of the integral in (2.32) to $a H_{\text {inf }}$. On the other hand, we write the lower limit of the integral as $k_{\mathrm{IR}}$, which in the case of inflationary magnetogenesis would correspond to the wave mode that exited the horizon at the beginning of inflation (and thus we note $k_{\mathrm{IR}} \ll a_{1} H_{\text {inf }}$ ). We see from (3.6) that the electric power spectrum is much larger than the magnetic power on super-horizon scales. Therefore we ignore the magnetic contributions to the energy density and thus obtain

$$
\rho_{\mathrm{EM}} \simeq \frac{4 \Gamma\left(s+\frac{1}{2}\right)^{2}}{\pi^{3}} H_{\mathrm{inf}}^{4} \int_{k_{\mathrm{IR}}}^{a H_{\mathrm{inf}}} \frac{d k}{k}\left(\frac{2 a H_{\mathrm{inf}}}{k}\right)^{2(s-2)}
$$

were we have extrapolated the super-horizon expression for $\mathcal{P}_{E}$ up to $k=a H_{\text {inf }}$. One can easily check that this energy density increases monotonically in time.

\subsection{2 $a>a_{1}$}

After the coupling $I$ becomes time independent, i.e. $a>a_{1}$, the mode function is a sum of plane waves,

$$
u_{k}=C_{+} e^{-i k\left(\tau-\tau_{1}\right)}+C_{-} e^{i k\left(\tau-\tau_{1}\right)}
$$


We obtain the constants $C_{+}$and $C_{-}$by matching (3.8) and its time-derivative with the approximate solutions (3.4), (3.5) at $a=a_{1}$.

For modes that are inside the horizon until $a=a_{1}$, i.e. $k \gg a_{1} H_{\text {inf }}$, the mode function stays as a positive frequency solution,

$$
C_{+} \simeq u_{k}\left(\tau_{1}\right) \simeq \frac{1}{(2 k)^{1 / 2} I_{1}} \exp \left\{i\left(\frac{k}{a_{1} H_{\mathrm{inf}}}+\frac{s-1}{2} \pi\right)\right\}, \quad C_{-} \simeq 0 .
$$

On the other hand for modes $k \ll a_{1} H_{\text {inf }}$, one finds

$$
C_{ \pm} \simeq e^{i\left(s-\frac{1}{2}\right) \pi} \frac{\Gamma\left(s+\frac{1}{2}\right)}{(8 \pi k)^{1 / 2} I_{1}}\left(\frac{k}{2 a_{1} H_{\mathrm{inf}}}\right)^{-s}\left\{ \pm 1+\frac{\pi}{\Gamma\left(s+\frac{1}{2}\right)^{2}}\left(\frac{k}{2 a_{1} H_{\mathrm{inf}}}\right)^{2 s}-\frac{i}{s-\frac{1}{2}} \frac{k}{2 a_{1} H_{\mathrm{inf}}}\right\} .
$$

Here in the \{\} parentheses we keep the terms whose amplitudes are much smaller than unity, as they can be important upon computing quantities such as $\left|u_{k}\right|$, or when further connecting to mode functions in later times. The time evolving $I$ has transformed $u_{k}$ outside the horizon into a mixture of positive and negative frequency solutions.

Thus the amplitudes of the electromagnetic fields are

$$
\begin{gathered}
\mathcal{P}_{B} \simeq \begin{cases}\frac{k^{4}}{2 \pi^{2} I_{1}^{2} a^{4}} \frac{4 s^{2} \Gamma\left(s-\frac{1}{2}\right)^{2}}{\pi}\left(\frac{2 a_{1} H_{\mathrm{inf}}}{k}\right)^{2(s-1)} & \text { for } k \ll a_{1} H_{\mathrm{inf}}, \\
\frac{k^{4}}{2 \pi^{2} I_{1}^{2} a^{4}} & \text { for } k \gg a_{1} H_{\mathrm{inf}},\end{cases} \\
\mathcal{P}_{E} \simeq \begin{cases}\frac{k^{4}}{2 \pi^{2} I_{1}^{2} a^{4}} \frac{\Gamma\left(s+\frac{1}{2}\right)^{2}}{\pi}\left(\frac{2 a_{1} H_{\mathrm{inf}}}{k}\right)^{2 s} & \text { for } k \ll a_{1} H_{\mathrm{inf}}, \\
\frac{k^{4}}{2 \pi^{2} I_{1}^{2} a^{4}} & \text { for } k \gg a_{1} H_{\mathrm{inf}} .\end{cases}
\end{gathered}
$$

Here we have shown the asymptotic forms in the limit of $a \gg a_{1}$. This is why the expression of $\mathcal{P}_{B}$ for $k \ll a_{1} H_{\text {inf }}$ extrapolated to $a=a_{1}$ differs from that in (3.6) by a factor of $4 s^{2}$. Comparing the magnetic power spectra between wave modes $k \lessgtr a_{1} H_{\text {inf }}$, the magnetic enhancement factor from inflationary magnetogenesis is obtained as

$$
\mathcal{A}_{\text {inf }}=\frac{4 s^{2} \Gamma\left(s-\frac{1}{2}\right)^{2}}{\pi}\left(\frac{2 a_{1} H_{\text {inf }}}{k}\right)^{2(s-1)} .
$$

For $s>1$, larger scales (i.e. smaller $k$ ) are more significantly enhanced as they exit the horizon earlier. On the other hand when $1 / 2<s<1$, the growing rate of the mode function decreases after exiting the horizon (cf. (3.4), (3.5)), and thus the magnetic field strength ends up being suppressed at large scales.

The electromagnetic energy density is obtained by integrating each of the above power spectra over the wave modes $k_{\mathrm{IR}}<k<a_{1} H_{\text {inf }}$ and $a_{1} H_{\text {inf }}<k<a H_{\text {inf }}$. In the limit of $a \gg a_{1}$, the energy density asymptotes to

$$
\rho_{\mathrm{EM}} \simeq \rho_{\mathrm{EM}}\left(\tau_{1}\right)\left(\frac{a_{1}}{a}\right)^{4}+\frac{H_{\mathrm{inf}}^{4}}{8 \pi^{2}}
$$

where $\rho_{\mathrm{EM}}\left(\tau_{1}\right)$ is obtained from (3.7). We now have a component that decays as $\propto a^{-4}$, plus a constant contribution of $\mathcal{O}\left(H_{\mathrm{inf}}^{4}\right)$ from modes within the range $a_{1} H_{\mathrm{inf}}<k<a H_{\mathrm{inf}}$. 


\subsection{Constraints on Inflation}

Using the above results, we now compute the amplitude of magnetic fields produced during inflation. Here we suppose that magnetogenesis happens only during inflation and the standard Maxwell theory is recovered at $a=a_{1}$. Then after magnetogenesis, the magnetic power spectrum decays as $a^{-4}$ until the present, cf. (2.41). Let us focus on a wave mode that exits the horizon before $a=a_{1}$, i.e. $k \ll a_{1} H_{\text {inf }}$, so that the magnetic fields on this scale are enhanced outside the horizon. The present amplitude of the magnetic field is

$$
\mathcal{P}_{B}\left(\tau_{0}, k\right)=4 s^{2} \mathcal{P}_{B}\left(\tau_{1}, k\right)\left(\frac{a_{1}}{a_{0}}\right)^{4},
$$

where we denote values in the present universe by the subscript 0 , and the factor $4 s^{2}$ arise due to the slight increase of $\left|u_{k}\right|$ soon after $a=a_{1}$, see discussions below (3.11).

The electromagnetic energy density monotonically increases until $a=a_{1}$ (cf. (3.7)), and then it starts to decrease. The inflation scale is restricted to satisfy $H_{\text {inf }} \ll M_{p}$ from observational constraints on inflationary gravitational waves, and thus the constant contribution of $\sim H_{\mathrm{inf}}^{4}$ to the energy density shown in (3.13) is much smaller than the total energy density of the inflating universe. Therefore, the electromagnetic energy density is always smaller than the background density if

$$
\rho_{\mathrm{EM}}\left(\tau_{1}\right)<3 M_{p} H_{\mathrm{inf}}^{2}
$$

is satisfied.

Let us now rewrite the present magnetic power spectrum in terms of the electric spectrum at time $\tau_{1}$ as

$$
\mathcal{P}_{B}\left(\tau_{0}, k\right)=\frac{s^{2}}{\left(s-\frac{1}{2}\right)^{2}} \frac{\mathcal{P}_{E}\left(\tau_{1}, k\right)}{H_{\mathrm{inf}}^{2}}\left(\frac{k}{a_{0}}\right)^{2}\left(\frac{a_{1}}{a_{\text {end }}}\right)^{2}\left(\frac{a_{\text {end }}}{a_{0}}\right)^{2},
$$

where $a_{\text {end }}$ is the scale factor at the end of inflation. We suppose that the universe is effectively matter-dominated after inflation until reheating, i.e.,

$$
\left(\frac{H_{\mathrm{reh}}}{H_{\mathrm{inf}}}\right)^{2}=\left(\frac{a_{\mathrm{end}}}{a_{\mathrm{reh}}}\right)^{3} .
$$

The subscript "reh" denotes quantities at reheating. The reheating scale $H_{\text {reh }}$ is related to the entropy density $s_{\text {reh }}$ as

$$
s_{\mathrm{reh}}=\frac{2 \pi^{2}}{45} g_{s *}\left(T_{\mathrm{reh}}\right)\left(\frac{90}{\pi^{2}} \frac{M_{p}^{2} H_{\mathrm{reh}}^{2}}{g_{*}\left(T_{\mathrm{reh}}\right)}\right)^{3 / 4},
$$

and by considering the entropy to be conserved after reheating, i.e. $s \propto a^{-3}$, the energy scale and redshift at reheating are related as

$$
\frac{H_{\mathrm{reh}}}{M_{p}} \approx 3 \times 10^{-63}\left(\frac{a_{0}}{a_{\mathrm{reh}}}\right)^{2} .
$$

Here we have chosen the relativistic degrees of freedom at reheating to be the maximum value allowed in the MSSM, $g_{*}=g_{s *}=228.75$. However we note that the numerical factor in (3.19) only depends 
weakly on the values of the relativistic degrees of freedom; e.g., $g_{*}=g_{s *}=10.75$ gives a factor 5 instead of 3 in the right hand side. Combining (3.17) and (3.19) gives

$$
\left(\frac{a_{\mathrm{end}}}{a_{0}}\right)^{2} \approx 3 \times 10^{-63}\left(\frac{H_{\mathrm{reh}}}{H_{\mathrm{inf}}}\right)^{1 / 3} \frac{M_{p}}{H_{\mathrm{inf}}} .
$$

Further using $1 G \approx 2 \times 10^{-20} \mathrm{GeV}^{2}$ (we use the Heaviside-Lorentz units) and $1 \mathrm{Mpc} \approx 2 \times 10^{29} \mathrm{eV}^{-1}$, the power spectrum (3.16) can be rewritten as

$$
\frac{\mathcal{P}_{B}\left(\tau_{0}, k\right)}{\left(10^{-15} \mathrm{G}\right)^{2}} \sim \frac{s^{2}}{\left(s-\frac{1}{2}\right)^{2}} \cdot \frac{I_{1}^{2}}{2} \frac{\mathcal{P}_{E}\left(\tau_{1}, k\right)}{3 M_{p}^{2} H_{\mathrm{inf}}^{2}} \cdot \frac{1}{I_{1}^{2}} \cdot\left(\frac{a_{1}}{a_{\mathrm{end}}}\right)^{2}\left(\frac{H_{\mathrm{reh}}}{H_{\mathrm{inf}}}\right)^{1 / 3}\left(\frac{k}{a_{0}} \mathrm{Mpc}\right)^{2} \frac{10^{-32} M_{p}}{H_{\mathrm{inf}}} .
$$

The factor $I_{1}^{2} \mathcal{P}_{E}\left(\tau_{1}, k\right) /\left(2 \cdot 3 M_{p}^{2} H_{\text {inf }}^{2}\right)$ denotes the energy density ratio at time $\tau_{1}$ between the electric field on the scale $k$ and the inflating background, cf. (2.32). This ratio should be smaller than unity to avoid strong backreaction from the electric field which can terminate magnetogenesis and/or inflation. Moreover, recall from the discussions below (2.1) that the coupling should satisfy $I_{1}^{2} \gtrsim 1$ in order to avoid strong couplings. Further noting that $a_{1} \leq a_{\text {end }}$ and $H_{\text {reh }} \leq H_{\text {inf }}$, we see from (3.21) that an extremely low scale inflation with

$$
H_{\text {inf }} \ll 10^{-32} M_{p} \sim 10^{-5} \mathrm{eV}
$$

is required in order to produce magnetic fields stronger than $10^{-15} \mathrm{G}$ on Mpc scales. ${ }^{4}$ It should also be noted that here we have discussed backreaction from electric fields with a certain wave mode $k$. Considering the energy density contributions from all super-horizon modes gives an even more stringent upper bound on $H_{\text {inf }}$. The difficulties with inflationary magnetogenesis have already been pointed out in previous studies, such as $[17,22] .{ }^{5}$ In particular, the work [22] derived a generic upper bound on $H_{\text {inf }}$ without specifying the time evolution of the mode function. Compared to their result, we arrived at a more stringent bound (3.22) because we have focused on the specific case where the coupling $I$ scales as a power-law of $a$.

The strong constraint on the inflation scale can be understood by the fact that the magnetic fields in the present universe traces back to possess much larger magnetic power in the earlier times, as they redshift as $B^{2} \propto a^{-4}$ after inflation. Thus a significant production of magnetic fields is required for inflationary magnetogenesis that happens at high energy scales. At the same time, higher inflation scales imply less cosmological time for magnetogenesis, in other words the electromagnetic vector potential needs to be more strongly enhanced in a shorter period time for higher inflation scales. Then the time-derivative of the vector potential would be so large that it sources significant electric fields whose backreaction on the inflating universe cannot be neglected. This observation leads us to seek magnetogenesis at lower energy scales, namely, in the post-inflationary universe. This will be the topic of the next section.

\footnotetext{
${ }^{4}$ At first glance, the right hand side of (3.21) appears to blow up as $s \rightarrow 1 / 2$. However, as we have already mentioned, the super-horizon approximation (3.5) cannot be trusted when $s$ is close to $1 / 2$. One can use other approximations that work well for $s=1 / 2$, and see that in such a case the magnetic fields cannot be significantly produced, independently of the inflation scale $H_{\mathrm{inf}}$.

${ }^{5}$ See also [37] which discusses magnetogenesis during significantly low scale inflation.
} 


\section{Magnetogenesis After Inflation}

We now turn to the investigation of magnetogenesis after inflation. We consider the post-inflationary universe to be dominated by an inflaton field that harmonically oscillates around its potential minimum, behaving like pressureless matter. The oscillating inflaton eventually decays into radiation, either perturbatively or nonperturbatively, and reheats the universe. During (p)reheating, the conductivity of the universe become very high, after which the electromagnetic vector potential becomes time independent and thus $B \propto a^{-2}, E=0$. Therefore we focus on the regime between the end of inflation and (p)reheating, during which the universe is cold and effectively matter-dominated.

\subsection{Evolution of Electromagnetic Fields}

The background universe under consideration is matter-dominated (MD), i.e. $H \propto a^{-3 / 2}, w=0$. As in the previous section, we study a coupling that scales as a power-law of $a$ during a finite period of time,

$$
I= \begin{cases}I_{1} & \text { for } a \leq a_{2}, \\ I_{1}\left(\frac{a_{2}}{a}\right)^{n} & \text { for } a_{2}<a \leq a_{3}, \\ I_{1}\left(\frac{a_{2}}{a_{3}}\right)^{n} \equiv I_{f} & \text { for } a>a_{3} .\end{cases}
$$

The coupling $I$ asymptotes to positive constant values $I_{1}$ and $I_{f}$ in the past and future, respectively, and the effective "horizon" of radius $|I / \dot{I}|=|n H|^{-1}$ emerges during $a_{2}<a<a_{3}$. Examples of scalar field couplings with such a behavior are presented in Section 6.2. We suppose the coupling to approach its final value $I_{f}$ before reheating, i.e., $a_{3}<a_{\text {reh }}$.

Under the time dependent $I$, the mode function on super-horizon scales is given in (2.38) with $\alpha=2 n+1 / 2$. We suppose the exponent $n$ to be positive, i.e.,

$$
n>0
$$

which gives rise to a growing mode $(a H)^{-4 n-1} \propto a^{2 n+\frac{1}{2}}$ for the super-horizon $u_{k}$.

\subsection{1 $a \leq a_{2}$}

The electromagnetic theory is initially the standard Maxwell theory, therefore the mode function is given by the plane wave solution as in (3.8),

$$
u_{k}=C_{+} e^{-i k\left(\tau-\tau_{1}\right)}+C_{-} e^{i k\left(\tau-\tau_{1}\right)} .
$$

We continue our discussions from the previous section where we studied inflationary magnetogenesis, and plug in their results as the initial conditions for the mode functions. The mode function for wave modes that underwent inflationary magnetogenesis (i.e. $k \ll a_{1} H_{\text {inf }}$ ) is given by (3.10), while it is simply the positive frequency solution (3.9) for modes $k \gg a_{1} H_{\text {inf }}$ which stayed inside the horizon during inflationary magnetogenesis.

We remark that, the case where inflationary magnetogenesis did not happen at all (or, the case where inflation itself did not happen) can be studied by simply taking $a_{1} \rightarrow 0$ in the following discussions, then all wave modes under consideration would satisfy $k \gg a_{1} H_{\text {inf }}$. 
Let us assume the wave modes that exited the effective/Hubble horizon during inflationary magnetogenesis to re-enter the Hubble horizon after reheating, i.e.,

$$
a_{1} H_{\mathrm{inf}} \ll a_{\mathrm{reh}} H_{\mathrm{reh}} .
$$

This assumption is adopted mainly to reduce clutter in the equations; it implies the relation $a_{1} H_{\text {inf }} \ll$ $a H$ during the post-inflationary epoch until reheating, which will allow us to drop many terms in the computations.

Focusing on super-horizon modes $k \ll a H$, the electromagnetic power spectra are

$$
\begin{gathered}
\mathcal{P}_{B} \simeq \begin{cases}\frac{k^{4}}{2 \pi^{2} I_{1}^{2} a^{4}} \frac{4 s^{2} \Gamma\left(s-\frac{1}{2}\right)^{2}}{\pi}\left(\frac{2 a_{1} H_{\mathrm{inf}}}{k}\right)^{2(s-1)}\left(1+\frac{2 s-1}{s} \frac{a_{1} H_{\mathrm{inf}}}{a H}\right)^{2} & \text { for } k \ll a_{1} H_{\mathrm{inf}}, \\
\frac{k^{4}}{2 \pi^{2} I_{1}^{2} a^{4}} & \text { for } k \gg a_{1} H_{\mathrm{inf}},\end{cases} \\
\mathcal{P}_{E} \simeq \begin{cases}\frac{k^{4}}{2 \pi^{2} I_{1}^{2} a^{4}} \frac{\Gamma\left(s+\frac{1}{2}\right)^{2}}{\pi}\left(\frac{2 a_{1} H_{\mathrm{inf}}}{k}\right)^{2 s} & \text { for } k \ll a_{1} H_{\mathrm{inf}}, \\
\frac{k^{4}}{2 \pi^{2} I_{1}^{2} a^{4}} & \text { for } k \gg a_{1} H_{\mathrm{inf}},\end{cases}
\end{gathered}
$$

which are the same as shown in (3.11), except for the magnetic fields on scales $k \ll a_{1} H_{\text {inf }}$. The difference arise due to the super-horizon mode function $u_{k}$ possessing a time evolving component $\propto(a H)^{-1}$ which is a (slowly) growing mode in the MD universe, cf. (2.38) and footnote 3. This component decays during inflation, and thus was neglected in (3.11). However it should be noted that this mode is always a subdominant component under the assumption of (4.4). We also remark that a purely positive (or negative) frequency solution gives a constant $\left|u_{k}\right|$, which is why $\mathcal{P}_{B}$ for modes $k \gg a_{1} H_{\text {inf }}$ have the same form during and after inflation.

Integrating the electromagnetic power spectra over super-horizon wave modes gives the energy density

$$
\rho_{\mathrm{EM}} \simeq \rho_{\mathrm{EM}}\left(\tau_{1}\right)\left(\frac{a_{1}}{a}\right)^{4}+\frac{H^{4}}{8 \pi^{2}},
$$

which takes the same form as in (3.13). The first term on the right hand side proportional to $\rho_{\mathrm{EM}}\left(\tau_{1}\right)$ denotes the electric density from wave modes that underwent inflationary magnetogenesis, i.e., $k_{\mathrm{IR}}<k<a_{1} H_{\text {inf }}$. The second term $\sim H^{4}$ represents the electromagnetic density mainly sourced from waved modes entering the horizon, $k \sim a H$.

\subsection{2 $a_{2}<a \leq a_{3}$}

The general solution after the effective "horizon" emerges is shown in (2.36) with $\alpha=2 n+1 / 2$. By matching its super-horizon approximation (2.38) with (4.3) at $a=a_{2}$, we obtain the mode function for wave numbers $k \ll a H$ as

$$
\begin{aligned}
u_{k} \simeq C_{+} e^{-i k\left(\tau_{2}-\tau_{1}\right)}\left[1+\frac{i}{2 n+\frac{1}{2}} \frac{k}{a_{2} H_{2}}\right. & \left.\left\{1-\left(\frac{a}{a_{2}}\right)^{2 n+\frac{1}{2}}\right\}\right] \\
+ & C_{-} e^{i k\left(\tau_{2}-\tau_{1}\right)}\left[1-\frac{i}{2 n+\frac{1}{2}} \frac{k}{a_{2} H_{2}}\left\{1-\left(\frac{a}{a_{2}}\right)^{2 n+\frac{1}{2}}\right\}\right],
\end{aligned}
$$


where the constants $C_{ \pm}$are given in (3.9) and (3.10), depending on whether $k \gtrless a_{1} H_{\text {inf }}$.

When $\left(a / a_{2}\right)^{2 n+\frac{1}{2}} \gg 1$, the electromagnetic power spectra are written as

$$
\begin{gathered}
\mathcal{P}_{B} \simeq \begin{cases}\frac{k^{4}}{2 \pi^{2} I_{1}^{2} a^{4}} \frac{4 s^{2} \Gamma\left(s-\frac{1}{2}\right)^{2}}{\pi}\left(\frac{2 a_{1} H_{\mathrm{inf}}}{k}\right)^{2(s-1)}\left\{1+\frac{2 s-1}{2 s\left(2 n+\frac{1}{2}\right)}\left(\frac{a}{a_{2}}\right)^{2 n} \frac{a_{1} H_{\mathrm{inf}}}{a H}\right\}^{2} \\
\frac{k^{4}}{2 \pi^{2} I_{1}^{2} a^{4}}\left\{1+\frac{1}{\left(2 n+\frac{1}{2}\right)^{2}}\left(\frac{a}{a_{2}}\right)^{4 n}\left(\frac{k}{a H}\right)^{2}\right\} & \text { for } k \ll a_{1} H_{\mathrm{inf}},\end{cases} \\
\mathcal{P}_{E} \simeq \begin{cases}\frac{k^{4}}{2 \pi^{2} I_{1}^{2} a^{4}}\left(\frac{a}{a_{2}}\right)^{4 n} \frac{\Gamma\left(s+\frac{1}{2}\right)^{2}}{\pi}\left(\frac{2 a_{1} H_{\mathrm{inf}}}{k}\right)^{2 s} & \text { for } k \gg a_{1} H_{\mathrm{inf}}, \\
\frac{k^{4}}{2 \pi^{2} I_{1}^{2} a^{4}}\left(\frac{a}{a_{2}}\right)^{4 n} & \text { for } k \ll a_{1} H_{\mathrm{inf}},\end{cases}
\end{gathered}
$$

Due to the time evolving $I$, the magnetic fields now have a component that scales as $B^{2} \propto a^{4 n-3}$ (given by the second terms in the \{\} parentheses), in addition to the normal component that decays as $B^{2} \propto a^{-4}$. The former component is initially negligible, however it can eventually dominate over the latter, depending on the duration of this period $a_{3} / a_{2}$. Hereafter, we suppose that the enhanced component $B^{2} \propto a^{4 n-3}$ eventually becomes dominant and assume

$$
\left(\frac{a_{3}}{a_{2}}\right)^{2 n} \frac{a_{1} H_{\mathrm{inf}}}{a_{3} H_{3}} \gg 1
$$

For modes $k \gg a_{1} H_{\text {inf }}$ which did not experience inflationary magnetogenesis (or in the case where inflationary magnetogenesis or inflation itself did not happen at all), the assumption (4.9) is translated into

$$
\left(\frac{a_{3}}{a_{2}}\right)^{2 n} \frac{k}{a_{3} H_{3}} \gg 1
$$

The electromagnetic energy density is enhanced by a factor $\left(a / a_{2}\right)^{2 n}$ during this period. From the power spectra above, one finds that the energy density takes the form

$$
\rho_{\mathrm{EM}} \simeq \rho_{\mathrm{EM}}\left(\tau_{1}\right)\left(\frac{a_{1}}{a}\right)^{4}\left(\frac{a}{a_{2}}\right)^{2 n}+\left\{\frac{1}{2}+\frac{4}{3(4 n+1)^{2}}\right\} \frac{H^{4}}{8 \pi^{2}}\left(\frac{a}{a_{2}}\right)^{2 n},
$$

as $\left(a / a_{2}\right)^{2 n}$ becomes much larger than unity.

\subsection{3 $a>a_{3}$}

In this final period before reheating, the Maxwell theory is again recovered and the mode function reduces to a sum of plane waves,

$$
u_{k}=\widetilde{C}_{+} e^{-i k\left(\tau-\tau_{3}\right)}+\widetilde{C}_{-} e^{i k\left(\tau-\tau_{3}\right)},
$$


whose constants $\widetilde{C}_{ \pm}$are found by matching the solutions (4.7) and (4.12) at $a=a_{3}$, giving

$$
\begin{aligned}
\widetilde{C}_{ \pm} \simeq \frac{C_{+}}{2} e^{-i k\left(\tau_{2}-\tau_{1}\right)}[1 & \left. \pm\left(\frac{a_{3}}{a_{2}}\right)^{2 n}+\frac{i}{2 n+\frac{1}{2}} \frac{k}{a_{2} H_{2}}\left\{1-\left(\frac{a_{3}}{a_{2}}\right)^{2 n+\frac{1}{2}}\right\}\right] \\
& +\frac{C_{-}}{2} e^{i k\left(\tau_{2}-\tau_{1}\right)}\left[1 \mp\left(\frac{a_{3}}{a_{2}}\right)^{2 n}-\frac{i}{2 n+\frac{1}{2}} \frac{k}{a_{2} H_{2}}\left\{1-\left(\frac{a_{3}}{a_{2}}\right)^{2 n+\frac{1}{2}}\right\}\right] .
\end{aligned}
$$

When $a \gg a_{3}$, the power spectra for the super-horizon modes $k \ll a H$ are

$$
\begin{aligned}
& \mathcal{P}_{B} \simeq \begin{cases}\frac{k^{4}}{2 \pi^{2} I_{f}^{2} a^{4}} \frac{16 \Gamma\left(s+\frac{1}{2}\right)^{2}}{\pi}\left(\frac{2 a_{1} H_{\mathrm{inf}}}{k}\right)^{2(s-1)}\left(\frac{a_{3}}{a_{2}}\right)^{2 n}\left(\frac{a_{1} H_{\mathrm{inf}}}{a H}\right)^{2} & \text { for } k \ll a_{1} H_{\mathrm{inf}}, \\
\frac{k^{4}}{2 \pi^{2} I_{f}^{2} a^{4}}\left(\frac{a_{3}}{a_{2}}\right)^{2 n}\left(\frac{2 k}{a H}\right)^{2} & \text { for } k \gg a_{1} H_{\mathrm{inf}},\end{cases} \\
& \mathcal{P}_{E} \simeq \begin{cases}\frac{k^{4}}{2 \pi^{2} I_{f}^{2} a^{4}}\left(\frac{a_{3}}{a_{2}}\right)^{2 n} \frac{\Gamma\left(s+\frac{1}{2}\right)^{2}}{\pi}\left(\frac{2 a_{1} H_{\mathrm{inf}}}{k}\right)^{2 s} & \text { for } k \ll a_{1} H_{\mathrm{inf}}, \\
\frac{k^{4}}{2 \pi^{2} I_{f}^{2} a^{4}}\left(\frac{a_{3}}{a_{2}}\right)^{2 n} & \text { for } k \gg a_{1} H_{\mathrm{inf}} .\end{cases}
\end{aligned}
$$

Note that here we have used the final value of the coupling $I_{f}$, cf. (4.1). Even for modes with $k \gg a_{1} H_{\text {inf }}$ which did not undergo magnetogenesis during inflation, the mode function $u_{k}$ has been transformed into a mixture of positive and frequency solutions during the post-inflationary evolution of $I$, and now $u_{k}$ is dominated by the growing mode $\propto a^{1 / 2}$.

Comparing $\mathcal{P}_{B}$ for modes $k \gg a_{1} H_{\text {inf }}$ with the power spectrum in the standard Maxwell theory (2.41) at reheating, the magnetic enhancement factor from post-inflationary magnetogenesis reads

$$
\mathcal{A}_{\text {post }}=\left(\frac{a_{3}}{a_{2}}\right)^{2 n}\left(\frac{2 k}{a_{\mathrm{reh}} H_{\mathrm{reh}}}\right)^{2} .
$$

We note that this enhancement is for wave modes that stay outside the Hubble (and effective) horizon throughout the post-inflationary magnetogenesis. In contrast to inflationary magnetogenesis (3.12), here the enhancement is stronger at smaller scales (larger $k$ ).

Magnetic fields on wave modes $k \ll a_{1} H_{\text {inf }}$ are enhanced both during and after inflation. The net enhancement factor from magnetogenesis in the two regimes is

$$
\begin{aligned}
\mathcal{A}_{\text {full }} & =\frac{16 \Gamma\left(s+\frac{1}{2}\right)^{2}}{\pi}\left(\frac{2 a_{1} H_{\mathrm{inf}}}{k}\right)^{2(s-1)}\left(\frac{a_{3}}{a_{2}}\right)^{2 n}\left(\frac{a_{1} H_{\mathrm{inf}}}{a_{\mathrm{reh}} H_{\mathrm{reh}}}\right)^{2} \\
& =\mathcal{A}_{\text {inf }} \mathcal{A}_{\text {post }}\left(\frac{2 s-1}{2 s}\right)^{2}\left(\frac{a_{1} H_{\mathrm{inf}}}{k}\right)^{2} .
\end{aligned}
$$

Interestingly the combination of inflationary and post-inflationary magnetogenesis gives rise to a much stronger enhancement than the simple product of the individual magnetogenesis scenarios.

Finally, the electromagnetic energy density when $a \gg a_{3}$ is

$$
\rho_{\mathrm{EM}} \simeq \rho_{\mathrm{EM}}\left(\tau_{1}\right)\left(\frac{a_{1}}{a}\right)^{4}\left(\frac{a_{3}}{a_{2}}\right)^{2 n}+\frac{11}{6} \frac{H^{4}}{8 \pi^{2}}\left(\frac{a_{3}}{a_{2}}\right)^{2 n} .
$$




\subsection{Constraints on Reheating}

In this subsection we analyze the magnetic fields produced from post-inflationary magnetogenesis alone. We will see that post-inflationary magnetogenesis is also strictly constrained by the strong coupling and backreaction problems, and that a significant production of large-scale magnetogenesis would require a reheating temperature that is extremely low. ${ }^{6}$

As is explained above (4.5), we can focus solely on post-inflationary magnetogenesis by taking $a_{1} \rightarrow 0$ and studying the wave modes $k \gg a_{1} H_{\text {inf }}$ in the above discussions. Hence for all wave modes we only need to consider the set of constants $\left|C_{+}\right|^{2}=1 / 2 k I_{1}^{2}, C_{-}=0$, as shown in (3.9).

Correspondingly, the electromagnetic energy densities (4.6), (4.11), and (4.17) ${ }^{7}$ only have the components proportional to $H^{4}$. In particular, the energy density ratio between the electromagnetic fields and the background universe scales as

$$
\frac{\rho_{\mathrm{EM}}}{3 M_{p}^{2} H^{2}} \simeq \begin{cases}\frac{H^{2}}{24 \pi^{2} M_{p}^{2}} \propto a^{-3} & \text { for } a \leq a_{2}, \\ \left(\frac{1}{2}+\frac{4}{3(4 n+1)^{2}}\right) \frac{H^{2}}{24 \pi^{2} M_{p}^{2}}\left(\frac{a}{a_{2}}\right)^{2 n} \propto a^{2 n-3} & \text { for } a_{2}<a \leq a_{3}, \\ \frac{11}{6} \frac{H^{2}}{24 \pi^{2} M_{p}^{2}}\left(\frac{a_{3}}{a_{2}}\right)^{2 n} \propto a^{-3} & \text { for } a_{3}<a .\end{cases}
$$

Considering a sub-Planckian universe, i.e. $H \ll M_{p}$, the electromagnetic backreaction can become significant only if $n>3 / 2$ so that the ratio increases during $a_{2}<a \leq a_{3}$. In other words, the backreaction is tiny throughout magnetogenesis if

$$
\frac{\rho_{\mathrm{EM}}\left(\tau_{3}\right)}{3 M_{p}^{2} H_{3}^{2}} \ll 1
$$

is satisfied.

We focus on scales that re-enter the Hubble horizon after reheating, i.e. $k \ll a_{\text {reh }} H_{\text {reh }}$, and also satisfy the condition (4.10) so that the magnetic fields are significantly enhanced on this scale. Then considering that the magnetic power after reheating scales as $\mathcal{P}_{B} \propto a^{-4}$, the present value reads

$$
\mathcal{P}_{B}\left(\tau_{0}, k\right)=\frac{k^{4}}{2 \pi^{2} I_{f}^{2} a_{\mathrm{reh}}^{4}}\left(\frac{a_{3}}{a_{2}}\right)^{2 n}\left(\frac{2 k}{a_{\mathrm{reh}} H_{\mathrm{reh}}}\right)^{2}\left(\frac{a_{\mathrm{reh}}}{a_{0}}\right)^{4} .
$$

Expressing $a_{\text {reh }}$ in terms of $H_{\text {reh }}$ using (3.19), and also from the second line of (4.18), we find

$$
\frac{\mathcal{P}_{B}\left(\tau_{0}, k\right)}{\left(10^{-15} \mathrm{G}\right)^{2}} \sim \frac{3(4 n+1)^{2}}{3(4 n+1)^{2}+8} \cdot \frac{\rho_{\mathrm{EM}}\left(\tau_{3}\right)}{3 M_{p}^{2} H_{3}^{2}} \cdot \frac{1}{I_{f}^{2}} \cdot\left(\frac{H_{\mathrm{reh}}}{H_{3}}\right)^{2}\left(\frac{k}{a_{0}} \mathrm{Mpc}\right)^{6}\left(\frac{10^{-23} \mathrm{MeV}}{H_{\mathrm{reh}}}\right)^{3} .
$$

Here, note that the condition (4.19) is required to avoid significant backreaction, $I_{f}^{2} \gtrsim 1$ to avoid strong couplings in the theory, and $H_{\text {reh }} \leq H_{3}$. Thus we see that requiring the generated magnetic

\footnotetext{
${ }^{6}$ Here we discuss reheating constraints on post-inflationary magnetogenesis, however we note that inflationary magnetogenesis can also impose constraints on reheating. See [25, 38].

${ }^{7}$ The magnetic power spectrum for small wave numbers that do not satisfy the condition (4.10) can be different from $\mathcal{P}_{B}$ for $k \gg a_{1} H_{\mathrm{inf}}$ shown in (4.14). However the contribution to the energy density from such small wave numbers is negligibly tiny, and so the result from (4.17), $\rho_{\mathrm{EM}} \simeq\left(11 H^{4} / 48 \pi^{2}\right)\left(a_{3} / a_{2}\right)^{2 n}$, is unaffected.
} 
fields to be stronger than $\sim 10^{-15} \mathrm{G}$ on Mpc scales bounds the reheating scale from above as

$$
H_{\text {reh }} \ll 10^{-23} \mathrm{MeV} \text {. }
$$

Such a low reheating scale is inconsistent with Big Bang Nucleosynthesis (BBN) which requires the reheating temperature to be at least about $5 \mathrm{MeV}[39$, 40, 41], i.e.,

$$
H_{\text {reh }} \gtrsim 10^{-20} \mathrm{MeV} \text {. }
$$

Thus we see that post-inflationary magnetogenesis alone cannot produce large magnetic fields on cosmological scales without having significant backreaction on the expanding universe, or going into the strong coupling regime. The magnetic enhancement is stronger for lower reheating scales, as is seen from the enhancement factor $\mathcal{A}_{\text {post }}$ in (4.15). Furthermore, the enhanced electromagnetic power spectra have larger amplitudes at smaller scales (larger $k$ ); the spectral indices are $d \ln \mathcal{P}_{B} / d \ln k=6$, $d \ln \mathcal{P}_{E} / d \ln k=4$, cf. (4.14). (This is in contrast to inflationary magnetogenesis which can produce red-tilted spectra.) As a consequence, the dominant contribution to the electromagnetic energy density comes from wave modes entering the horizon, i.e. $k^{-1}=(a H)^{-1}$. Since the comoving Hubble radius $(a H)^{-1}$ grows in an MD universe, post-inflationary magnetogenesis is pushed to later times in order to avoid the backreaction issue, and we have seen that this conflicts with BBN.

Before ending this section we should remark that, even though we have been trying to avoid electromagnetic backreaction, their actual effects on the MD universe is unclear. The work [18] studied a model of inflationary magnetogenesis and showed that electromagnetic backreaction significantly suppresses the production of magnetic fields during inflation. It would be interesting to examine whether backreaction is actually disastrous for post-inflation magnetogenesis as well.

\section{Large Magnetic Fields from Two Step Magnetogenesis}

We have seen in the previous sections the difficulties of the individual inflationary and post-inflationary magnetogenesis. Inflationary magnetogenesis imposed a severe upper bound on the inflation scale, while post-inflationary magnetogenesis could not be accomplished without spoiling BBN.

In this section we show that efficient production of large-scale magnetic fields can be achieved by combining the inflationary and post-inflationary magnetogenesis. This is possible due to the magnetogenesis in the two epochs working in different ways, for instance in terms of the scale dependencies of the produced electromagnetic spectra. One advantage of the two step magnetogenesis is that, the post-inflationary magnetic enhancement relaxes the generation of electric as well as magnetic fields during inflation. Another advantage is that, since inflationary magnetogenesis is typically more effective at larger scales (cf. (3.12)), it can compensate for the small scale (though super-horizon) electromagnetic fields intensively produced from post-inflationary magnetogenesis. Therefore the inflationary and post-inflationary magnetogenesis complement each other to evade the backreaction problem. Moreover, as we have seen in (4.16), post-inflation magnetogenesis works even more effectively for wave modes that has already underwent inflationary magnetogenesis.

\subsection{Magnetic Fields and Energy Bounds}

The basic formulae are already laid out in the previous sections, however a few things are worth noting. For wave modes that experience magnetic enhancement both during and after inflation, i.e. 
$k \ll a_{1} H_{\text {inf }}$, the magnetic power in the present universe is obtained by using (4.14) as

$$
\begin{aligned}
\mathcal{P}_{B}\left(\tau_{0}, k\right) & =\mathcal{P}_{B}\left(\tau_{\mathrm{reh}}, k\right)\left(\frac{a_{\mathrm{reh}}}{a_{0}}\right)^{4} \\
& =\frac{k^{4}}{2 \pi^{2} I_{f}^{2} a_{\mathrm{reh}}^{4}} \frac{16 \Gamma\left(s+\frac{1}{2}\right)^{2}}{\pi}\left(\frac{2 a_{1} H_{\mathrm{inf}}}{k}\right)^{2(s-1)}\left(\frac{a_{3}}{a_{2}}\right)^{2 n}\left(\frac{a_{1} H_{\mathrm{inf}}}{a_{\mathrm{reh}} H_{\mathrm{reh}}}\right)^{2}\left(\frac{a_{\mathrm{reh}}}{a_{0}}\right)^{4} .
\end{aligned}
$$

We can further rewrite the expression using (3.19) as

$$
\frac{\mathcal{P}_{B}\left(\tau_{0}, k\right)}{\left(10^{-15} \mathrm{G}\right)^{2}} \sim 10^{-94}\left(\frac{2 a_{1} H_{\mathrm{inf}}}{k}\right)^{2 s}\left(\frac{a_{3}}{a_{2}}\right)^{2 n} \frac{\Gamma\left(s+\frac{1}{2}\right)^{2}}{I_{f}^{2}}\left(\frac{k}{a_{0}} \mathrm{Mpc}\right)^{6} \frac{10^{-20} \mathrm{MeV}}{H_{\mathrm{reh}}} .
$$

Considering $I_{f}^{2} \gtrsim 1$ and the BBN constraint (4.23), one sees that $B \sim 10^{-15} \mathrm{G}$ on Mpc scales can be produced if the combined inflationary/post-inflationary effect gives $\left(2 a_{1} H_{\text {inf }} / k\right)^{2 s}\left(a_{3} / a_{2}\right)^{2 n} \gtrsim 10^{94}$.

As we have seen in the previous sections, the electromagnetic energy density basically consists of two components which are respectively proportional to $\rho_{\mathrm{EM}}\left(\tau_{1}\right)$ and $H^{4}$, each multiplied by some powers of the scale factor. (We already discussed the $H^{4}$ component in Section 4.2, and the electromagnetic density during inflation in Section 3.2.) The energy density ratio between the electromagnetic fields and the background $\rho_{\mathrm{EM}} / 3 M_{p}^{2} H^{2}$ grows monotonically during $a<a_{1}$, while

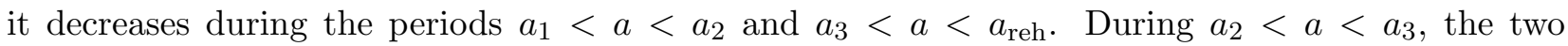
components of $\rho_{\text {EM }}$ may either grow or decay, depending on the value of the exponent $n$. Therefore we find that the electromagnetic backreaction is always tiny if the energy ratio is small at times $\tau_{1}$ and $\tau_{3}$, i.e.,

$$
\begin{aligned}
\frac{\rho_{\mathrm{EM}}\left(\tau_{1}\right)}{3 M_{p}^{2} H_{\mathrm{inf}}^{2}} & \ll 1, \\
\frac{\rho_{\mathrm{EM}}\left(\tau_{3}\right)}{3 M_{p}^{2} H_{3}^{2}} & \ll 1 .
\end{aligned}
$$

The energy condition at $\tau_{1}(5.3)$ constrains the magnetic field amplitude in a similar fashion as in (3.21), except for that now we have an extra enhancement for the magnetic amplitude of

$$
\frac{\mathcal{A}_{\text {full }}}{\mathcal{A}_{\text {inf }}}=\mathcal{A}_{\text {post }}\left(\frac{2 s-1}{2 s}\right)^{2}\left(\frac{a_{1} H_{\mathrm{inf}}}{k}\right)^{2}=\left(\frac{2 s-1}{s}\right)^{2}\left(\frac{a_{3}}{a_{2}}\right)^{2 n}\left(\frac{a_{1} H_{\mathrm{inf}}}{a_{\mathrm{reh}} H_{\mathrm{reh}}}\right)^{2},
$$

which arise from the post-inflationary magnetogenesis. This extra term can significantly weaken the bound (3.22) on the inflation scale.

The bound at $\tau_{3}$ (5.4) consists of two conditions, namely,

$$
\begin{gathered}
\left(\frac{1}{2}+\frac{4}{3(4 n+1)^{2}}\right) \frac{H_{3}^{2}}{24 \pi^{2} M_{p}^{2}}\left(\frac{a_{3}}{a_{2}}\right)^{2 n} \ll 1, \\
\frac{\rho_{\mathrm{EM}}\left(\tau_{1}\right)}{3 M_{p}^{2} H_{3}^{2}}\left(\frac{a_{1}}{a_{3}}\right)^{4}\left(\frac{a_{3}}{a_{2}}\right)^{2 n} \ll 1 .
\end{gathered}
$$

The former condition (5.6) restricts the magnetic fields as in (4.21), but now with an extra factor of

$$
\frac{\mathcal{A}_{\text {full }}}{\mathcal{A}_{\text {post }}}=\mathcal{A}_{\text {inf }}\left(\frac{2 s-1}{2 s}\right)^{2}\left(\frac{a_{1} H_{\text {inf }}}{k}\right)^{2}=\frac{\Gamma\left(s+\frac{1}{2}\right)^{2}}{\pi}\left(\frac{2 a_{1} H_{\text {inf }}}{k}\right)^{2 s}
$$


in the right hand side. The extra enhancement due to the inflationary magnetogenesis weakens the constraint (4.22) on reheating.

The latter bound (5.7) is a constraint that arise only in the combined case of inflationary and post-inflationary magnetogenesis; it represents the constraint on large-scale electric fields that were produced during inflation, and further enhanced by the post-inflationary magnetogenesis. Focusing on this bound, the magnetic power (5.1) can be rewritten as (recall that $\rho_{\mathrm{EM}}\left(\tau_{1}\right)$ can be obtained by taking $a=a_{1}$ in $\left.(3.7)\right){ }^{8}$

$$
\begin{aligned}
\frac{\mathcal{P}_{B}\left(\tau_{0}, k\right)}{\left(10^{-15} \mathrm{G}\right)^{2}} \sim 2(s-2)\{1- & \left.\left(\frac{k_{\mathrm{IR}}}{a_{1} H_{\mathrm{inf}}}\right)^{2(s-2)}\right\}^{-1}\left(\frac{k_{\mathrm{IR}}}{k}\right)^{2(s-2)} \\
& \cdot \frac{\rho_{\mathrm{EM}}\left(\tau_{1}\right)}{3 M_{p}^{2} H_{3}^{2}}\left(\frac{a_{1}}{a_{3}}\right)^{4}\left(\frac{a_{3}}{a_{2}}\right)^{2 n} \cdot \frac{1}{I_{f}^{2}} \cdot \frac{a_{3}}{a_{\mathrm{reh}}}\left(\frac{k}{a_{0}} \mathrm{Mpc}\right)^{2} \frac{10^{-10} \mathrm{MeV}}{H_{\mathrm{reh}}} .
\end{aligned}
$$

Since we are interested in wave modes lying in the range $k_{\mathrm{IR}} \ll k \ll a_{1} H_{\text {inf }}$, the first line of the right hand side is smaller than unity. The right hand side is also suppressed by the energy condition (5.7), the requirement $I_{f}^{2} \gtrsim 1$ for avoiding strong coupling, and $a_{3}<a_{\text {reh. }}$ In order to produce $B \sim 10^{-15} \mathrm{G}$ on Mpc scales, the reheating scale $H_{\text {reh }}$ should be smaller than $\sim 10^{-10} \mathrm{MeV}$, or in terms of the reheating temperature less than a few hundred $\mathrm{GeV}$. Thus the reheating scale cannot be arbitrary high, however we stress that the bound is now significantly weakened compared to the case of postinflation magnetogenesis alone (4.22), and that the two step magnetogenesis can be completed before BBN.

\subsection{Case Study}

Let us now demonstrate that the two step magnetogenesis can actually produce large magnetic fields, even with high scale inflation. We examine the evolution of the electromagnetic spectra under a fixed set of parameters that allow efficient magnetogenesis.

The example parameters are chosen as follows: We set the inflation scale to $H_{\text {inf }}=10^{-6} M_{p} \sim$ $10^{12} \mathrm{GeV}$, and the reheating scale at $H_{\text {reh }}=10^{-18} \mathrm{MeV}$ (which corresponds to $T_{\text {reh }} \approx 50 \mathrm{MeV}$ in terms of temperature). The infrared cutoff of the energy density integral (3.7), corresponding to the wave mode that exits the horizon at the beginning of inflation, is set to $k_{\mathrm{IR}} / a_{0}=10^{-2} H_{0} \sim$ $10^{-6} \mathrm{Mpc}^{-1}$, i.e., during inflation the wave mode $k_{\mathrm{IR}}$ exited the horizon about 5 e-foldings before the mode corresponding to the present Hubble radius did.

The coupling $I$ scales with $s=5 / 2$ during inflation as in (3.1), $n=6$ after inflation as (4.1), then settles down to its final value $I_{f}=1$. We set the scale factor $a_{1}$ by $a_{1} H_{\text {inf }} / a_{0}=10^{4} \mathrm{Mpc}^{-1}$, which by using (3.20) can be rephrased as the time evolution of $I$ to first terminate at roughly 30 e-foldings before the end of inflation. The second phase of $I$ evolution in the post-inflation universe lasts for

\footnotetext{
${ }^{8}$ In obtaining the right hand side of $(5.10)$, we have assumed $s \neq 2$. The case with $s=2$ is obtained by simply replacing the terms in the first line by

$$
2(s-2)\left\{1-\left(\frac{k_{\mathrm{IR}}}{a_{1} H_{\mathrm{inf}}}\right)^{2(s-2)}\right\}^{-1} \longrightarrow\left\{\ln \left(\frac{a_{1} H_{\mathrm{inf}}}{k_{\mathrm{IR}}}\right)\right\}^{-1} .
$$


a period of $a_{3} / a_{2} \approx 1.4 \times 10^{6}$, where $a_{3}$ is related to the scale factor at reheating by $a_{\text {reh }} / a_{3}=10$. In terms of the energy scale, these corresponds to $H_{2} \sim 10^{-7} \mathrm{MeV}$ and $H_{3} \sim 10^{-17} \mathrm{MeV}$.

This set of parameters gives magnetic fields of $\mathcal{P}_{B}\left(\tau_{0}, k\right)^{1 / 2} \sim 10^{-15} \mathrm{G}$ on scales $k / a_{0} \sim 1 \mathrm{Mpc}^{-1}$ (which is easily seen from the formula (5.2)), while satisfying the energy bounds (5.3) and (5.4) as $\rho_{\mathrm{EM}}\left(\tau_{1}\right) / 3 M_{p}^{2} H_{\mathrm{inf}}^{2} \sim 10^{-3}, \rho_{\mathrm{EM}}\left(\tau_{3}\right) / 3 M_{p}^{2} H_{3}^{2} \sim 10^{-2}$.

In Figure 1 we plot the electromagnetic power spectra at different times. The blue solid lines denote the magnetic power spectra $\mathcal{P}_{B}^{1 / 2}$, and the red dashed lines are the electric power $\mathcal{P}_{E}^{1 / 2}$. Upon plotting the spectra, we have set the mode function $u_{k}$ as the positive frequency solution (3.3) during $a<a_{1}$, and then used the exact solution for $u_{k}$ (2.36) at each epoch. The results of course match with the approximate expressions presented in the previous sections, except for at intermediate scales such as $k \sim a_{1} H_{\text {inf }}$, where we switch between different limits used in the approximations. The dashed vertical lines in the figures represent the wave mode $k=a_{1} H_{\text {inf }}$, while the solid vertical lines in Figures $1(a)$ and $1(d)$ show the Hubble radius, i.e. $k=a H$. In Figures $1(b)$ and $1(c)$, the displayed wave modes are all outside the horizon.

Figure 1(a) shows the electromagnetic power spectra at $a=10^{-2} a_{1}$. Inflationary magnetogenesis lifts the spectra on super-horizon scales; as is shown in (3.6), the magnetic power spectrum for superhorizon modes scales as $\mathcal{P}_{B}^{1 / 2} \propto k^{3-s}$, and the electric fields have a redder spectrum with $\mathcal{P}_{E}^{1 / 2} \propto k^{2-s}$. On the other hand for sub-horizon modes, the magnetic and electric spectra are identical, scaling as $\mathcal{P}^{1 / 2} \propto k^{2}$. The wave number where the spectra bends, i.e. $k \sim a H_{\text {inf }}$, shifts towards larger $k$ modes as inflation proceeds.

Between the two phases of $I$ evolution, i.e. $a_{1}<a<a_{2}$, the electromagnetic power spectra conserve their shapes; the uplifted spectra on large scales (small $k$ ) connect to the blue spectra on small scales at $k \sim a_{1} H_{\text {inf }}$, cf. (3.11) and (4.5). This is seen in Figure 1(b) which shows the spectra at $a \approx 3 \times 10^{8} a_{1}\left(<a_{\text {end }}\right)$.

Figure 1 (c) shows the spectra at $a \approx 2 a_{2}\left(<a_{3}\right)$. The post-inflationary magnetogenesis during $a_{2}<a<a_{3}$ enhances the electric fields more strongly than the magnetic fields (see (4.8)), and thus the two spectra no longer overlap. The magnetic power now has a term that scales as $\mathcal{P}_{B}^{1 / 2} \propto k^{3}$, and this component dominates the spectrum at small scales.

When the coupling $I$ has approached its final constant value $I_{f}$, i.e. $a>a_{3}$, the magnetic spectrum $\mathcal{P}_{B}^{1 / 2}$ for wave modes in the range $a_{1} H_{\text {inf }} \ll k \ll a H$ scales as $k^{3}$, while it remains to be $\propto k^{3-s}$ at larger scales $k \ll a_{1} H_{\text {inf }}$, cf. (4.14). The spectra right before reheating $a=a_{\text {reh }}$ is shown in Figure 1(d). The $k$ modes close to the right edge of the plot have already re-entered the Hubble horizon, and thus the power spectra exhibit oscillatory behaviors.

We also plot the electromagnetic power as a function of $\ln a$ in Figure 2, focusing on the scale $k / a_{0}=1 \mathrm{Mpc}^{-1}$. Note that this wave mode exits the Hubble horizon during inflationary magnetogenesis (i.e. while $a<a_{1}$ ), and re-enters the horizon after reheating is completed. During the first phase of magnetogenesis, the magnetic field initially increases as $B \propto a^{s-2}$, then after the wave mode exits the horizon as $a^{2 s-3}$. While the coupling $I$ stays constant the magnetic amplitude redshifts as $a^{-2}$, but soon after $I$ again becomes time dependent, $B$ starts to evolve as $a^{2 n-3 / 2}$. After the standard Maxwell theory is recovered at $a=a_{3}$, the magnetic field redshifts as $B \propto a^{-3 / 2}$, and after 


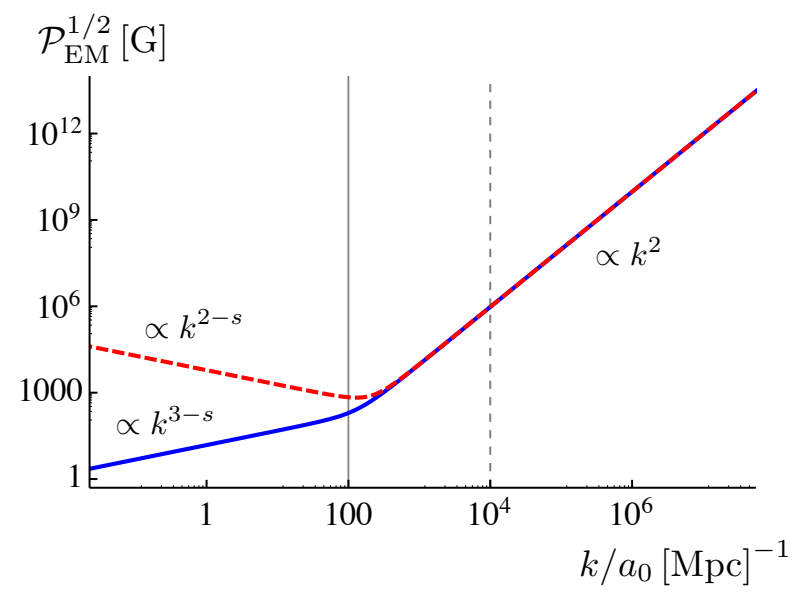

(a) $a=10^{-2} a_{1}$

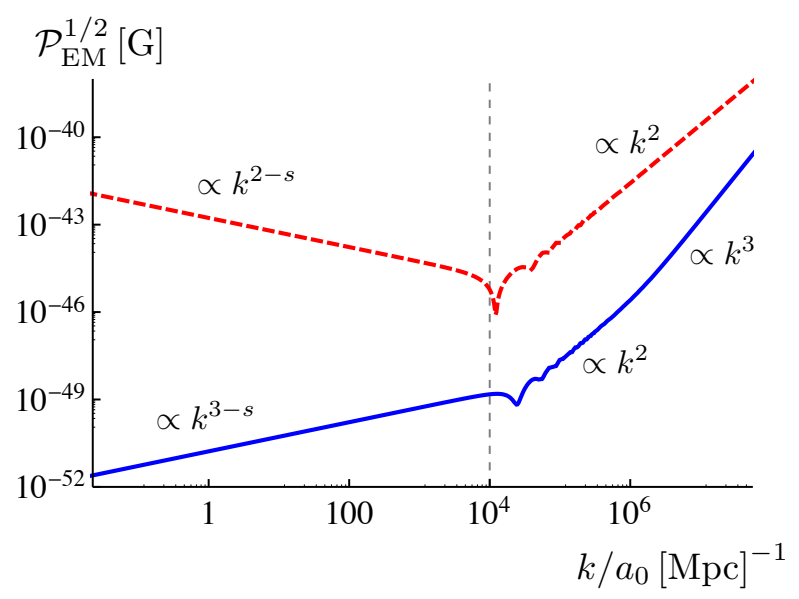

(c) $a \approx 2 a_{2}\left(<a_{3}\right)$

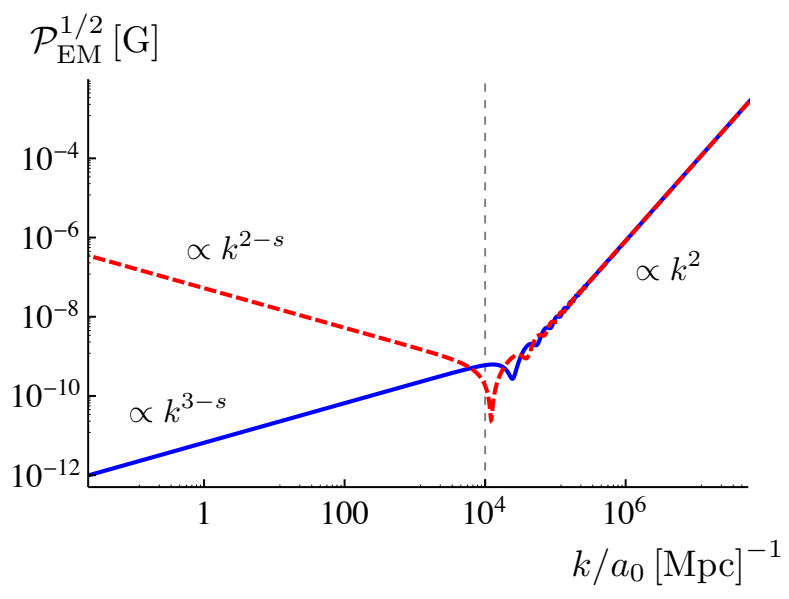

(b) $a \approx 3 \times 10^{8} a_{1}\left(<a_{\text {end }}\right)$

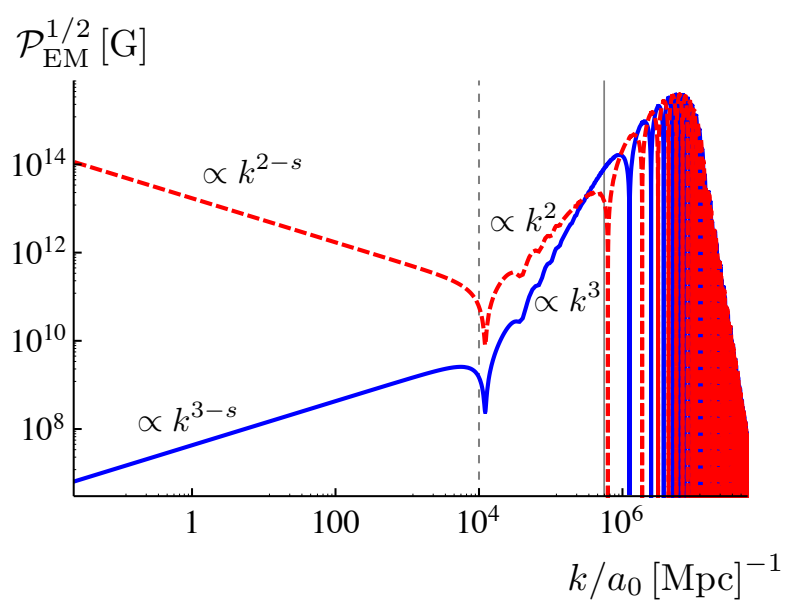

(d) $a=a_{\text {reh }}$

Figure 1: Electromagnetic power spectra at different times. $\mathcal{P}_{B}^{1 / 2}$ is shown as the blue solid lines, and $\mathcal{P}_{E}^{1 / 2}$ by the red dashed lines. The solid vertical lines denote the Hubble radius at each time (i.e. $k=a H$ ), while the dashed vertical lines represent the wave mode $k=a_{1} H_{\mathrm{inf}}$.

reheating as $a^{-2}$. For the chosen set of parameters, the generated magnetic field in the present universe has an amplitude of $B \sim 10^{-15} \mathrm{G}$ on Mpc scales. The evolution of the electric fields is similar to that of the magnetic fields, but with larger amplitude during most of the expansion history. The electric power spectrum is not shown beyond $a=a_{\mathrm{reh}}$, as the conductivity of the universe becomes high during reheating and the electric fields vanish.

In Figure 3, the time evolution of the electromagnetic energy density is shown up until reheating. The blue solid line denotes the magnetic contribution (see (2.32)), and the red dashed line is from the electric fields. The two lines lie on top of each other in the time between the two periods of magnetogenesis. We have also plotted the background density $3 M_{p}^{2} H^{2}$ shown as the black dotted line. As we have discussed in Section 5.1, the energy density ratio $\rho_{\mathrm{EM}} / 3 M_{p}^{2} H^{2}$ peaks at $a=a_{1}$ and $a=a_{3}$, taking values of order $10^{-3}$ and $10^{-2}$ respectively, under the parameter set chosen in 


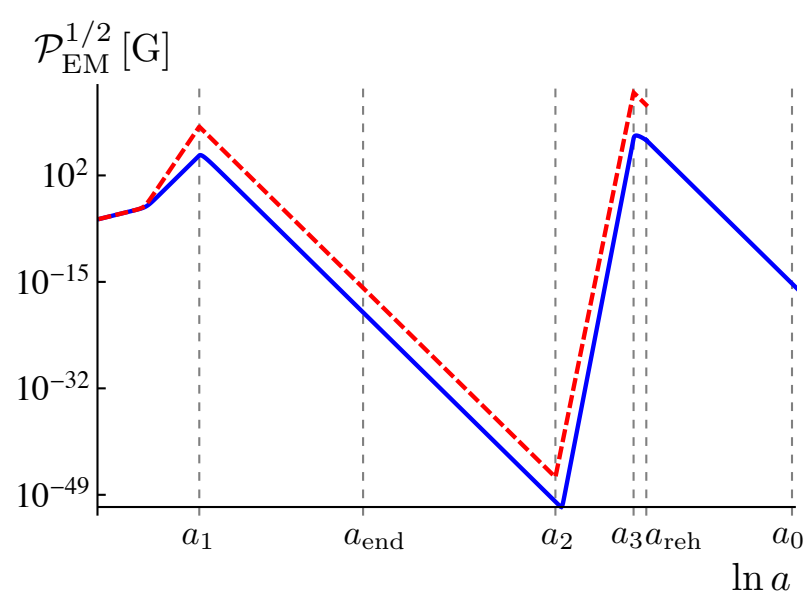

Figure 2: Time evolution of the electromagnetic power spectra for $k / a_{0}=1 \mathrm{Mpc}^{-1} \cdot \mathcal{P}_{B}^{1 / 2}$ : blue solid, $\mathcal{P}_{E}^{1 / 2}$ : red dashed.

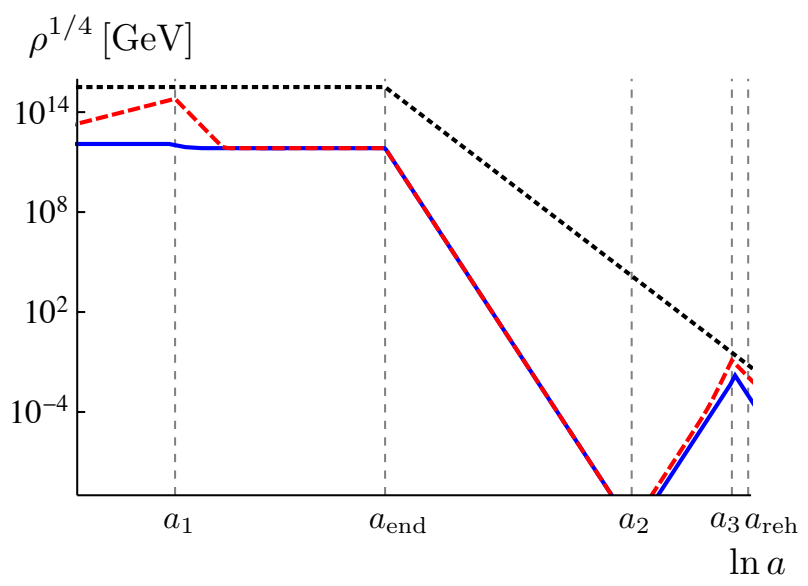

Figure 3: Time evolution of energy densities. Magnetic energy density: blue solid, electric density: red dashed, background density: black dotted.

this section. In the figure, the electric density appears to come close to the background density, especially at $a=a_{3}$. However it should be noted that we have plotted $\rho^{1 / 4}$, and that the units of the vertical axis vary by many orders of magnitude.

Thus we have seen that the combined inflationary/post-inflationary magnetogenesis can efficiently produce large-scale magnetic fields, as the two phases of magnetogenesis help each other to avoid the electromagnetic density from dominating the universe. The post-inflation magnetic enhancement relaxes inflationary magnetogenesis, therefore ameliorates the electric backreaction problem during inflation. At the same time, inflationary magnetogenesis can lift the magnetic spectrum at large scales (small $k$ ), and therefore can reduce the overall electromagnetic spectrum while maintaining magnetic power at large scales. This feature helps the post-inflation magnetogenesis which alone would produce too large electromagnetic power at small scales. On the other hand, the two step magnetogenesis produces large-scale electric power which may dominate the post-inflationary universe, and this has lead to a new constraint (5.7). However we have seen through the example case studied in this section that such electric densities can be put under control.

As for the dynamical coupling $I$, it either monotonically decreases in time or stays constant until it approaches the final value $I_{f}=1$. Therefore the interactions between the electromagnetic fields and other sectors such as charged fermions are strongly suppressed through most of the history of the universe.

\section{Examples of Scalar Couplings}

Let us show some examples of scalar field couplings that realize the time dependent $I$ considered in the previous sections. We individually discuss the dynamical couplings during and after inflation, but they can be combined to drive the two step magnetogenesis. We focus on couplings with scalar 
fields, however it should be noted that non-minimal couplings to gravity could also play a similar role.

Some of the examples in the following are somewhat ad hoc as they are designed to reproduce the simplified behaviors for $I$ in (3.1) and (4.1). It would be interesting to start from simple forms of scalar couplings and then discuss magnetogenesis that follows.

We should also remark that in explicitly constructed models, the produced electromagnetic fields can backreact on the coupled scalar. Furthermore, the electromagnetic fields may decay into the scalar particles. To know whether such effects become important requires a full treatment of the combined scalar/electromagnetic dynamics. We leave this for future work.

\subsection{Couplings During Inflation}

The dynamical coupling that behaves as (3.1) during inflation can be realized by the inflaton field, or some other spectator field.

\subsubsection{Inflaton Field}

Let us consider an inflaton field $\phi$ with a quadratic potential

$$
V(\phi)=\frac{1}{2} m^{2} \phi^{2}
$$

Then one can solve the slow-roll approximations $3 H \dot{\phi} \simeq-m^{2} \phi, 3 M_{p}^{2} H^{2} \simeq m^{2} \phi^{2} / 2$, and obtain

$$
\phi^{2}-\phi_{1}^{2} \simeq-4 M_{p}^{2} \ln \frac{a}{a_{1}} .
$$

Here $a=a_{1}$ corresponds to the time when the inflaton field $\phi$ crosses $\pm \phi_{1}$. As was discussed in previous works such as [16], by considering an exponential coupling to the electromagnetic kinetic term of the form

$$
I(\phi)=I_{1}\left\{1+\exp \left(\frac{s}{4} \frac{\phi^{2}-\phi_{1}^{2}}{M_{p}^{2}}\right)\right\}
$$

one sees that it evolves as

$$
I \simeq I_{1}\left\{1+\left(\frac{a_{1}}{a}\right)^{s}\right\} .
$$

If $s>0$, then the coupling $I$ scales as $a^{-s}$ when $\phi^{2}>\phi_{1}^{2}$, and then asymptotes to the constant

value $I_{1}$ for $\phi^{2}<\phi_{1}^{2}$. Therefore (3.1) is realized. The discussion here can be generalized to arbitrary power-law inflaton potentials [16].

\subsubsection{Spectator Field}

One can also imagine a case where the electromagnetic fields are coupled to a scalar that has minimal effect on the inflationary background. Such a spectator field $\sigma$ can also source the dynamical coupling. Suppose that $\sigma$ has a quadratic potential

$$
V(\sigma)=\frac{1}{2} m^{2} \sigma^{2}
$$


with a mass $m$ much lighter than the inflationary Hubble $H_{\text {inf }}$. Then the slow-roll equation $3 H_{\text {inf }} \dot{\sigma} \simeq$ $-m^{2} \sigma$ is solved as

$$
\sigma \propto a^{-m^{2} / 3 H_{\mathrm{inf}}^{2}}
$$

In this case, a polynomial coupling of the form

$$
I(\sigma)=I_{1}\left\{1+\left(\frac{\sigma}{\sigma_{1}}\right)^{3 s H_{\mathrm{inf}}^{2} / m^{2}}\right\}
$$

evolves as (6.4). However we should also remark that in this example, $s$ of order unity requires the exponent $3 s H_{\mathrm{inf}}^{2} / m^{2}$ in (6.7) is to be much larger than unity.

\subsection{Couplings After Inflation}

The coupling (4.1) which was discussed for post-inflationary magnetogenesis can be realized by rolling scalars, or oscillating scalars.

\subsubsection{Rolling Scalars}

Let us again consider a spectator scalar field $\sigma$ with a quadratic potential

$$
V(\sigma)=\frac{1}{2} m^{2} \sigma^{2} .
$$

We suppose that the background universe is effectively matter-dominated, and that the energy density of $\sigma$ is tiny compared to the total energy of the universe. If the field's mass is lighter than the Hubble parameter, i.e. $m^{2} \ll H^{2}$, then a homogeneous $\sigma$ field follows an attractor solution [42, 43]

$$
\frac{9}{2} H \dot{\sigma} \simeq-m^{2} \sigma
$$

Note that this is similar to the slow-roll approximation during inflation, except for that the numerical factor in the left hand side is $9 / 2$ in an MD universe. (6.9) can be solved using $H^{2} \propto a^{-3}$, giving

$$
\ln \left(\frac{\sigma}{\sigma_{2}}\right) \simeq \frac{2}{27} \frac{m^{2}}{H_{2}^{2}}\left\{1-\left(\frac{a}{a_{2}}\right)^{3}\right\},
$$

where the subscript 2 denotes quantities measured at a certain time. Therefore, considering a coupling of the form

$$
I(\sigma)=I_{f}\left[1+\left\{\ln \left(\frac{\sigma_{3}}{\sigma_{2}}\right)^{2}\right\}^{n / 3}\left\{\ln \left(\frac{\sigma}{\sigma_{2}}\right)^{2}-\frac{8}{27} \frac{m^{2}}{H_{2}^{2}}\right\}^{-n / 3}\right]
$$

where the subscript 3 is for quantities at some time after $t_{2}$, then one sees that the coupling is approximated by

$$
I \simeq I_{f}\left[1+\left\{\left(\frac{a_{3}}{a_{2}}\right)^{3}-1\right\}^{n / 3}\left\{\left(\frac{a}{a_{2}}\right)^{3}+1\right\}^{-n / 3}\right] .
$$

This coupling reproduces the behavior of (4.1) for $n>0$. 


\subsubsection{Oscillating Scalars}

A scalar $\sigma$ with a quadratic potential (6.8) eventually starts to oscillate when the Hubble parameter becomes comparable to its mass. The harmonically oscillating field acts like pressureless dust, and so the field's oscillation amplitude $\widetilde{\sigma}$ decays as $\propto a^{-3 / 2}$. Such an oscillating field can also source the dynamical coupling.

Let us consider a coupling as follows,

$$
I(\sigma)=I_{f}\left(1+\left|\frac{\sigma}{\widetilde{\sigma}_{3}}\right|^{2 n / 3}\right),
$$

where we suppose the constants $n$ and $\widetilde{\sigma}_{3}$ to be positive, and that $|\sigma|$ initially takes a field value much larger than $\widetilde{\sigma}_{3}$. Prior to the $\sigma$ oscillation, i.e. $H \gg m$, the coupling $I$ only slowly evolves. But once the field starts to oscillate, $I$ decays as $\propto a^{-n}$ when averaging over the oscillations. As the oscillation amplitude $\widetilde{\sigma}$ becomes smaller than $\widetilde{\sigma}_{3}$, the coupling $I$ asymptotes to a constant value $I_{f}$. This appears to reproduce the time dependent behavior (4.1) that was discussed for post-inflationary magnetogenesis.

However it should be noted that during the $\sigma$ oscillations, the coupling (6.13) also oscillates about $I_{f}$. Each time $\sigma$ crosses its origin $\sigma=0$, the change in the effective frequency of the mode function $u_{k}$ may become nonadiabatic (see (2.13)), leading to resonant amplifications of the electromagnetic fields. Investigation of such effects goes beyond the scope of this paper, but it would be interesting to explore the possibility of producing magnetic fields from parametric resonance. ${ }^{9}$

A model without parametric resonance may be realized by having multiple oscillating fields. For example, considering a complex scalar field $\Phi$ possessing a potential along the phase direction such as

$$
V(\Phi)=m^{2}|\Phi|^{2}+\left(\lambda \frac{\Phi^{c}}{M^{c-4}}+\text { h.c. }\right)
$$

then $\Phi$ can keep rotating in the complex plane without crossing the origin. With a coupling that depends on the decaying radial component,

$$
I(\Phi)=I_{f}\left(1+\left|\frac{\Phi}{\widetilde{\Phi}_{3}}\right|^{2 n / 3}\right)
$$

the mode function $u_{k}$ may evolve adiabatically.

\section{Conclusions}

In this work, we explored cosmological magnetogenesis during the two phases when the universe is cold: the inflationary epoch, and the post-inflationary epoch prior to reheating, during which the universe is dominated by the oscillating inflaton. Magnetogenesis in each phase alone are highly constrained by the strong coupling and backreaction problems, however, we have found that the

\footnotetext{
${ }^{9}$ However we also note that, even if resonant amplifications of magnetic fields happen during inflation, it would not improve much the situation for inflationary magnetogenesis alone. This is because a rather strict upper bound on the inflation scale can still be obtained independently of how the mode function evolves during inflation, see [22].
} 
combined inflationary and post-inflationary magnetogenesis can overcome the difficulties and efficiently produce large-scale magnetic fields. In particular, we demonstrated that the combined inflationary/post-inflationary magnetogenesis scenario can produce magnetic fields stronger than $10^{-15} \mathrm{G}$ on Mpc scales without running into the strong coupling regime, or producing too large electric fields that would dominate the universe. The proposed model is compatible even with high scale inflation.

The strong enhancement of the magnetic fields is made possible in the two step scenario due to the magnetogenesis in the two epochs working in very different ways. The magnetic enhancement in the post-inflationary universe reduces the need for a significant production of magnetic fields during inflation, and thus relaxes the constraints on inflationary magnetogenesis, including those from the backreaction problem and the excessive production of cosmological density perturbations. On the other hand, inflationary magnetogenesis enhances large-scale magnetic fields and thus can relatively suppress the small-scale (but super-horizon) electromagnetic fields produced during postinflationary magnetogenesis. Therefore the two phases of magnetogenesis mutually prevent the electromagnetic fields from dominating the universe in each epoch, while maintaining magnetic power at large scales. Moreover, we have shown that the net magnetic enhancement from the two step magnetogenesis is much stronger than a naive product of the enhancements from the individual phases of magnetogenesis.

In order to generate large magnetic fields from the cosmological background, we considered breaking the conformal invariance of the Maxwell theory $-I^{2} F_{\mu \nu} F^{\mu \nu} / 4$ through a time dependent coupling $I$ that scales as a power-law of the scale factor. As we discussed in Section 6, such a dynamical $I$ can arise from couplings with the inflaton or some other spectator field(s) that rolls along its effective potential, or oscillates about the potential minimum. Here we note that the produced electromagnetic fields may backreact on the directly coupled scalars in some cases. To see whether such effects become important requires a detailed analysis of explicitly constructed models. We leave this for future work.

The difficulty of post-inflationary magnetogenesis alone was presented in the form of the strict upper bound on the reheating scale $H_{\text {reh }} \ll 10^{-23} \mathrm{MeV}(4.22)$, which is incompatible with BBN. Upon deriving this bound we have assumed the scaling $I \propto a^{-n}$, and so the generality of the reheating bound remains an open question. For example, electromagnetic couplings with oscillating scalars can lead to further amplification of the magnetic fields through parametric resonance, which may allow the post-inflationary universe alone to create large magnetic fields, without the aid of inflationary magnetogenesis. Thus it would be interesting to derive a generic bound on post-inflationary magnetogenesis without specifying how the electromagnetic mode function evolves in time. Such an analysis was carried out for inflationary magnetogenesis in [22], and it may be possible to apply their discussions to the post-inflationary universe as well. We also note that the magnetic enhancement in the post-inflation universe should be affected by the details of the reheating process. We have assumed the conductivity of the universe to suddenly become high towards the end of the inflatondominated epoch, however the conductivity may start to gradually increase from earlier times if the oscillating inflaton decays perturbatively. On the other hand, preheating $[44,45]$ may lead to a sudden growth of the conductivity.

Although more detailed work will be required to verify whether there actually exists intergalactic magnetic fields, investigation of cosmological magnetic fields may provide an observational window 
into the very early universe. We demonstrated that large-scale magnetic fields can actually be created from the cosmological background. We hope that our mechanism will provide new insights into explaining our magnetized universe from the cosmological point of view.

\section{Acknowledgements}

It is a pleasure to thank Niayesh Afshordi, Avery Broderick, Carlos Palenzuela, Richard Shaw, and Chris Thompson for very useful discussions. I am also grateful to Shinji Mukohyama for useful discussions as well as helpful comments on a draft.

\section{References}

[1] F. Tavecchio, G. Ghisellini, L. Foschini, G. Bonnoli, G. Ghirlanda and P. Coppi, Mon. Not. Roy. Astron. Soc. 406, L70 (2010) [arXiv:1004.1329 [astro-ph.CO]].

[2] A. Neronov and I. Vovk, Science 328, 73 (2010) [arXiv:1006.3504 [astro-ph.HE]].

[3] S. 'i. Ando and A. Kusenko, Astrophys. J. 722, L39 (2010) [arXiv:1005.1924 [astro-ph.HE]].

[4] A. M. Taylor, I. Vovk and A. Neronov, Astron. Astrophys. 529, A144 (2011) [arXiv:1101.0932 [astro-ph.HE]].

[5] K. Takahashi, M. Mori, K. Ichiki, S. Inoue and H. Takami, Astrophys. J. 771, L42 (2013).

[6] J. Finke, L. Reyes and M. Georganopoulos, arXiv:1303.5093 [astro-ph.HE].

[7] A. E. Broderick, P. Chang and C. Pfrommer, Astrophys. J. 752, 22 (2012) [arXiv:1106.5494 [astro-ph.CO]].

[8] F. Miniati and A. Elyiv, Astrophys. J. 770, 54 (2013) [arXiv:1208.1761 [astro-ph.CO]].

[9] M. S. Turner and L. M. Widrow, Phys. Rev. D 37, 2743 (1988).

[10] B. Ratra, Astrophys. J. 391, L1 (1992).

[11] W. D. Garretson, G. B. Field and S. M. Carroll, Phys. Rev. D 46, 5346 (1992) [hep-ph/9209238].

[12] M. Gasperini, M. Giovannini and G. Veneziano, Phys. Rev. Lett. 75, 3796 (1995) [hepth/9504083].

[13] M. Giovannini and M. E. Shaposhnikov, Phys. Rev. D 62, 103512 (2000) [hep-ph/0004269].

[14] A. -C. Davis, K. Dimopoulos, T. Prokopec and O. Tornkvist, Phys. Lett. B 501, 165 (2001) [Phys. Rev. Focus 10, STORY9 (2002)] [astro-ph/0007214].

[15] K. Bamba and J. Yokoyama, Phys. Rev. D 69, 043507 (2004) [astro-ph/0310824].

[16] J. Martin and J. 'i. Yokoyama, JCAP 0801, 025 (2008) [arXiv:0711.4307 [astro-ph]]. 
[17] V. Demozzi, V. Mukhanov and H. Rubinstein, JCAP 0908, 025 (2009) [arXiv:0907.1030 [astroph.CO]].

[18] S. Kanno, J. Soda and M. -a. Watanabe, JCAP 0912, 009 (2009) [arXiv:0908.3509 [astroph.CO]].

[19] T. Vachaspati, Phys. Lett. B 265, 258 (1991).

[20] J. M. Cornwall, Phys. Rev. D 56, 6146 (1997) [hep-th/9704022].

[21] T. Vachaspati, Phil. Trans. Roy. Soc. Lond. A 366, 2915 (2008) [arXiv:0802.1533 [astro-ph]].

[22] T. Fujita and S. Mukohyama, JCAP 1210, 034 (2012) [arXiv:1205.5031 [astro-ph.CO]].

[23] T. Suyama and J. 'i. Yokoyama, Phys. Rev. D 86 (2012) 023512 [arXiv:1204.3976 [astro-ph.CO]].

[24] M. Giovannini, Phys. Rev. D 87, no. 8, 083004 (2013) [arXiv:1302.2243 [hep-th]].

[25] C. Ringeval, T. Suyama and J. 'i. Yokoyama, JCAP 1309, 020 (2013) [arXiv:1302.6013 [astroph.CO]].

[26] S. Nurmi and M. S. Sloth, arXiv:1312.4946 [astro-ph.CO].

[27] T. Fujita and S. Yokoyama, arXiv:1402.0596 [astro-ph.CO].

[28] J. R. Shaw and A. Lewis, Phys. Rev. D 86, 043510 (2012) [arXiv:1006.4242 [astro-ph.CO]].

[29] D. G. Yamazaki, T. Kajino, G. J. Mathew and K. Ichiki, Phys. Rept. 517, 141 (2012) [arXiv:1204.3669 [astro-ph.CO]].

[30] S. Camera, C. Fedeli and L. Moscardini, JCAP03(2014)027 [arXiv:1311.6383 [astro-ph.CO]].

[31] P. Berger, A. Kehagias and A. Riotto, arXiv:1402.1044 [astro-ph.CO].

[32] L. M. Widrow, Rev. Mod. Phys. 74, 775 (2002) [astro-ph/0207240].

[33] J. D. Barrow, R. Maartens and C. G. Tsagas, Phys. Rept. 449, 131 (2007) [astro-ph/0611537].

[34] A. Kandus, K. E. Kunze and C. G. Tsagas, Phys. Rept. 505, 1 (2011) [arXiv:1007.3891 [astroph.CO]].

[35] G. Dvali, O. Pujolas and M. Redi, Phys. Rev. D 76, 044028 (2007) [hep-th/0702117 [HEP-TH]].

[36] B. Himmetoglu, C. R. Contaldi and M. Peloso, Phys. Rev. D 80, 123530 (2009) [arXiv:0909.3524 [astro-ph.CO]].

[37] R. J. Z. Ferreira, R. K. Jain and M. S. Sloth, JCAP 1310, 004 (2013) [arXiv:1305.7151 [astroph.CO]].

[38] V. Demozzi and C. Ringeval, JCAP 1205, 009 (2012) [arXiv:1202.3022 [astro-ph.CO]].

[39] M. Kawasaki, K. Kohri and N. Sugiyama, Phys. Rev. Lett. 82, 4168 (1999) [astro-ph/9811437]. 
[40] M. Kawasaki, K. Kohri and N. Sugiyama, Phys. Rev. D 62, 023506 (2000) [astro-ph/0002127].

[41] S. Hannestad, Phys. Rev. D 70, 043506 (2004) [astro-ph/0403291].

[42] T. Chiba, Phys. Rev. D 79, 083517 (2009) [Erratum-ibid. D 80, 109902 (2009)] [arXiv:0902.4037 [astro-ph.CO]].

[43] M. Kawasaki, T. Kobayashi and F. Takahashi, Phys. Rev. D 84, 123506 (2011) [arXiv:1107.6011 [astro-ph.CO]].

[44] L. Kofman, A. D. Linde and A. A. Starobinsky, Phys. Rev. Lett. 73, 3195 (1994) [hepth/9405187].

[45] L. Kofman, A. D. Linde and A. A. Starobinsky, Phys. Rev. D 56, 3258 (1997) [hep-ph/9704452]. 